\title{
Semi-classical states for the nonlinear Choquard equations: existence, multiplicity and concentration at a potential well
}

\author{
Silvia Cingolani \\ Dipartimento di Matematica \\ Università degli Studi di Bari Aldo Moro \\ Via E. Orabona 4, 70125 Bari, Italy \\ Kazunaga Tanaka \\ Department of Mathematics \\ School of Science and Engineering \\ Waseda University \\ 3-4-1 Ohkubo, Shijuku-ku, Tokyo 169-8555, Japan
}

\begin{abstract}
We study existence and multiplicity of semi-classical states for the nonlinear Choquard equation:

$$
-\varepsilon^{2} \Delta v+V(x) v=\frac{1}{\varepsilon^{\alpha}}\left(I_{\alpha} * F(v)\right) f(v) \text { in } \mathbb{R}^{N},
$$

where $N \geq 3, \alpha \in(0, N), I_{\alpha}(x)=\frac{A_{\alpha}}{|x|^{N-\alpha}}$ is the Riesz potential, $F \in C^{1}(\mathbb{R}, \mathbb{R}), F^{\prime}(s)=f(s)$ and $\varepsilon>0$ is a small parameter.

We develop a new variational approach and we show the existence of a family of solutions concentrating, as $\varepsilon \rightarrow 0$, to a local minima of $V(x)$ under general conditions on $F(s)$. Our result is new also for $f(s)=|s|^{p-2} s$ and applicable for $p \in\left(\frac{N+\alpha}{N}, \frac{N+\alpha}{N-2}\right)$. Especially, we can give the existence result for locally sublinear case $p \in\left(\frac{N+\alpha}{N}, 2\right)$, which gives a positive answer to an open problem arisen in recent works of Moroz and Van Schaftingen.
\end{abstract}


We also study the multiplicity of positive single-peak solutions and we show the existence of at least $\operatorname{cupl}(K)+1$ solutions concentrating around $K$ as $\varepsilon \rightarrow 0$, where $K \subset \Omega$ is the set of minima of $V(x)$ in a bounded potential well $\Omega$, that is, $m_{0} \equiv \inf _{x \in \Omega} V(x)<\inf _{x \in \partial \Omega} V(x)$ and $K=\left\{x \in \Omega ; V(x)=m_{0}\right\}$.

Keywords: Nonlinear Choquard equation, semiclassical states, non-local nonlinearities, positive solutions, potential well, relative cup-length

AMS Subject Classification: 35Q55, 35Q40, 35J20, 58E05

\section{Introduction}

In this paper we consider the nonlocal equation

$$
-\varepsilon^{2} \Delta v+V(x) v=\frac{1}{\varepsilon^{\alpha}}\left(I_{\alpha} * F(v)\right) f(v) \text { in } \mathbb{R}^{N}, \quad v>0, \quad v \in H^{1}\left(\mathbb{R}^{N}\right),
$$

where $\varepsilon>0$ is a positive parameter, $N \geq 3, \alpha \in(0, N), I_{\alpha}: \mathbb{R}^{N} \backslash\{0\} \rightarrow \mathbb{R}$ the Riesz potential defined by

$$
I_{\alpha}(x)=\frac{\Gamma\left(\frac{N-\alpha}{2}\right)}{\Gamma\left(\frac{\alpha}{2}\right) \pi^{N / 2} 2^{\alpha}|x|^{N-\alpha}}
$$

and $F(s) \in C^{1}(\mathbb{R}, \mathbb{R})$ and $f(s)=F^{\prime}(s)$.

In 1954 the equation (1.1) with $N=3, \alpha=2$ and $F(s)=\frac{1}{2}|s|^{2}$ was introduced by Pekar in [41] to describe the quantum theory of a polaron at rest and in 1976 it was arisen in the work of Choquard on the modeling of an electron trapped in its own hole, in a certain approximation to Hartree Fock theory of one-component plasma [30] (see also [25, 26]). In particular the equation (1.1) with $V \equiv 1$ corresponds to the stationary nonlinear Hartree equation. Indeed if $v$ is a solution of (1.1), then the wave function $\psi(x, t)=$ $e^{i t / \varepsilon} v(x)$ is a solitary wave of the time-dependent Hartree equation

$$
\varepsilon i \psi_{t}=-\varepsilon^{2} \Delta \psi-\frac{1}{\varepsilon^{2}}\left(\frac{1}{|x|} *|\psi|^{2}\right) \psi \quad \text { in } \mathbb{R} \times \mathbb{R}^{3} .
$$

Finally, we recall that the three-dimensional nonlocal equation was also proposed by Penrose [42, 43, 44, 35] in his discussion on the self-gravitational collapse of a quantum mechanical wave-function and in that context it is 
known as the Schrödinger-Newton equation. Indeed, after a suitable change of variable, it can be written down as

$$
\left\{\begin{array}{l}
-\varepsilon^{2} \Delta \phi+V(x) \phi=U \phi, \\
-\Delta U=4 \pi \gamma|\phi|^{2}
\end{array}\right.
$$

where $\phi: \mathbb{R}^{3} \rightarrow \mathbb{C}, U: \mathbb{R}^{3} \rightarrow \mathbb{R}$ are unknown functions.

In literature the nonlocal equation (1.1) is usually called the nonlinear Choquard equation.

In the present paper we are interested to the search of semi-classical states for the nonlinear Choquard equation, namely solutions to (1.1) which exist for small values of $\varepsilon$ and have a specific behavior as $\varepsilon$ tends to 0 . We recall that the semiclassical analysis for nonlinear Schrödinger equations has been largely investigated in the last years, starting from the pioneering paper of Floer and Weistein [22] (see also [1, 21, 40]).

Concerning the nonlocal case, Wei and Winter [49] established the existence of a family of solutions of the Schrödinger-Newton equations (1.3), concentrating as $\varepsilon \rightarrow 0$ around a non-degenerate critical point of the potential $V(x)$ when inf $V(x)>0$ via a Lyapunov-Schmidt reduction method (see also [45] when $\liminf V(x)|x|^{\gamma}>0$ for some $\gamma \in[0,1)$ ). We note that the Lyapunov-Schmidt reduction method relies on the existence and uniqueness (up to translation) of the positive ground state solution of the limit problem:

$$
-\Delta u+u=\left(\frac{1}{|x|} *|u|^{2}\right) u \quad \text { in } \mathbb{R}^{3}
$$

which is established by Lieb in [30] (see also [29, 33]). Successively in [14, 19 ] variational methods were employed to study the semiclassical analysis for the three-dimensional magnetic Choquard equation with $\alpha=2$ and $F(s)=\frac{1}{2}|s|^{2}$.

In this work by means of a new approach we study the nonlinear Choquard equation (1.1), where $\alpha \in(0, N)$ and $f(s)$ is a general nonlinearity which does not satisfy a monotonicity assumption nor Ambrosetti-Rabinowitz type condition [2]. Defining $u(x)=v(\varepsilon x)$, the equation (1.1) is equivalent to

$$
-\Delta u+V(\varepsilon x) u=\left(I_{\alpha} * F(u)\right) f(u) \text { in } \mathbb{R}^{N}, u>0, u \in H^{1}\left(\mathbb{R}^{N}\right) .
$$

Thus, we try to find critical points of the corresponding functional:

$J_{\varepsilon}(u)=\frac{1}{2} \int_{\mathbb{R}^{N}}|\nabla u|^{2}+V(\varepsilon x) u^{2} d x-\frac{1}{2} \int_{\mathbb{R}^{N}}\left(I_{\alpha} * F(u)\right) F(u) d x: H^{1}\left(\mathbb{R}^{N}\right) \rightarrow \mathbb{R}$. 
It is natural to ask the existence of a family $\left(u_{\varepsilon}\right)$ of solutions of (1.5), which concentrates to a local minima of $V(x)$ as $\varepsilon$ tends to 0 .

To give our main result, we assume that

(f1) $f \in C(\mathbb{R}, \mathbb{R})$;

(f2) there exists $C>0$ such that for every $s \in \mathbb{R}$,

$$
|s f(s)| \leq C\left(|s|^{\frac{N+\alpha}{N}}+|s|^{\frac{N+\alpha}{N-2}}\right)
$$

$F(s)=\int_{0}^{s} f(t) d t$ satisfies

$$
\lim _{s \rightarrow 0} \frac{F(s)}{|s|^{\frac{N+\alpha}{N}}}=0, \quad \lim _{s \rightarrow+\infty} \frac{F(s)}{|s|^{\frac{N+\alpha}{N-2}}}=0
$$

(f4) there exists $s_{0} \in \mathbb{R}, s_{0} \neq 0$ such that $F\left(s_{0}\right)>0$.

(f5) $f$ is odd and $f$ is positive on $(0,+\infty)$.

We remark that the conditions (f1)-(f5) are in the spirit of Berestycki and Lions (see [3, 27, 37]) and cover the case $f(s)=|s|^{p-2} s$ with $p \in\left(\frac{N+\alpha}{N}, \frac{N+\alpha}{N-2}\right)$. For $V(x)$, we assume

(V1) $V \in C\left(\mathbb{R}^{N}, \mathbb{R}\right), \inf _{x \in \mathbb{R}^{N}} V(x) \equiv \underline{V}>0, \sup _{x \in \mathbb{R}^{N}} V(x)<\infty$.

(V2) There is a bounded domain $\Omega \subset \mathbb{R}^{N}$ such that

$$
m_{0} \equiv \inf _{x \in \Omega} V(x)<\inf _{x \in \partial \Omega} V(x)
$$

We also set

$$
K=\left\{x \in \Omega ; V(x)=m_{0}\right\}
$$

For $x_{0} \in K$, we have a formal limit problem:

$$
-\Delta U+m_{0} U=\left(I_{\alpha} * F(U)\right) f(U) \text { in } \mathbb{R}^{N}, U>0, U \in H^{1}\left(\mathbb{R}^{N}\right),
$$

whose solutions are characterized as critical points of

$$
L_{m_{0}}(u)=\frac{1}{2} \int_{\mathbb{R}^{N}}|\nabla u|^{2}+m_{0} u^{2} d x-\frac{1}{2} \int_{\mathbb{R}^{N}}\left(I_{\alpha} * F(u)\right) F(u) d x .
$$


The nonlinear Choquard equation (1.8) has been introduced and studied by Ma and Zhao in [34] and successively in [36, 37] (see also the recent survey [39]). In particular, in [37, Moroz and Van Schaftingen proved that under (f1)-(f5) the limiting equation (1.8) has a positive ground state solution, which is radially symmetric about some point (see also [13, 20]).

However, differently from (1.4), we do not have, in general, uniqueness and non-degeneracy for the ground state solutions of the limit equation (1.8) and it seems difficult to perform the Lyapunov-Schmidt reduction method for deriving semiclassical states of (1.5).

In [38], Moroz and Van Schaftingen proved existence of a single-peak solution for (1.1) concentrating at a local minima of $V$, when $f(s)=|s|^{p-2} s$, $p \in\left[2, \frac{N+\alpha}{N-2}\right)$ using a novel nonlocal penalization technique, and recently in [48] Yang, Zhang and Zhang showed the existence under (f4), $\lim _{t \rightarrow \infty} \frac{f(t)}{t^{\frac{\alpha+2}{N-2}}}=$ 0 and

$$
\lim _{t \rightarrow 0^{+}} \frac{f(t)}{t}=0
$$

They also proved the existence of multi-peak solutions, whose each peak concentrates at different local minimum point of $V(x)$ as $\varepsilon \rightarrow 0$. Thus, for a singular perturbation problem, the situation is more delicate and stronger conditions on the behavior of $f(s)$ as $s \sim 0$ are required in [38, 48].

We improve these results. Our main result is

Theorem 1.1 Suppose $N \geq 3$, (f1)-(f5), (V1)-(V2) and let $K$ be given in (1.7). Then (1.1) has at least one positive solution concentrating in $K$ as $\varepsilon \rightarrow 0$.

As a special case, we have

Corollary 1.2 Suppose $N \geq 3$ and $f(s)=|s|^{p-2} s$ with $p \in\left(\frac{N+\alpha}{N}, \frac{N+\alpha}{N-2}\right)$. Moreover assume (V1)-(V2). Then (1.1) has has at least one positive solution concentrating in $K$ as $\varepsilon \rightarrow 0$.

Remark 1.3 (i) Our Theorem 1.1 and Corollary 1.2 can be applied to the locally sublinear case, e.g., $f(s)=|s|^{p-2} s, p \in\left(\frac{N+\alpha}{N}, 2\right)$. In particular, it gives a positive answer to an open problem arisen in Moroz and Van Schaftingen [38, Section 6.1]. 
(ii) For $f(s)=|s|^{p-2} s$, the range $\left(\frac{N+\alpha}{N}, \frac{N+\alpha}{N-2}\right)$ of $p$ is optimal for the existence of nontrivial solutions. In fact, in [36. Theorem 2]. Moroz and Van Schaftingen showed that there are not nontrivial solutions outside.

To show our Theorem 1.1 and Corollary 1.2, we develop a new variational argument which relies and extends some works by Byeon and Jeanjean [9] and Byeon and Tanaka [10, 11. We remark that in [9, 10, 11] they deal with singular perturbation problems for nonlinear Schrödinger equations, while our argument also works for the non-local problem (1.1).

Let $E\left(m_{0}\right)$ be the least energy level for the limit problem:

$$
E\left(m_{0}\right)=\inf \left\{L_{m_{0}}(\omega) ; \omega \in H^{1}\left(\mathbb{R}^{N}\right) \backslash\{0\}, L_{m_{0}}^{\prime}(\omega)=0\right\}
$$

and we set for $s_{0} \in\left(0, \frac{1}{2}\right)$

$$
\begin{aligned}
S_{m_{0}}= & \left\{\omega \in H^{1}\left(\mathbb{R}^{N}\right) \backslash\{0\} ; L_{m_{0}}^{\prime}(\omega)=0, L_{m_{0}}(\omega)=E\left(m_{0}\right),\right. \\
& \left.\omega(0)=\max _{x \in \mathbb{R}^{N}} \omega(x)\right\} \\
\widehat{S}= & \left\{\omega(x / s) ; \omega \in S_{m_{0}}, s \in\left[1-s_{0}, 1+s_{0}\right]\right\} .
\end{aligned}
$$

We note that $S_{m_{0}}$ is compact. We also set for $\nu>0$ small

$$
N_{\varepsilon}(\nu)=\left\{u(x)=U(x)+\varphi(x) ; U \in \widehat{S},\|\varphi\|_{H^{1}}<\nu, \varepsilon \beta(u) \in K_{d_{0}}\right\},
$$

where $\beta(u)$ is a center of mass of $u$, defined in Section 3 and $K_{d_{0}}$ is a neighborhood of $K$ and we try to find critical points of $J_{\varepsilon}(u)$ in $N_{\varepsilon}(\nu)$. We note that the parameter $s$ is introduced corresponding to the scaling property of $L_{m_{0}}(u)$ and our definition of $\widehat{S}, N_{\varepsilon}(\nu)$ is slightly different from those in [9, 10, 11].

Such a localized variational method is used together with a penalization method in [9, 15, 16] or a tail minimizing operator in [10, 11] to ensure that concentration of solutions occurs in the desired region. In [9, 15, 16, it is introduced a penalization term and first a critical point of the penalized functional is found.

In [10, 11], for a given $u \in N_{\varepsilon}(\nu)$ it is considered a minimizing problem of type:

$$
\begin{array}{ll}
\operatorname{minimize}\left\{J_{\varepsilon}(v) ; \quad\right. & v \in H^{1}\left(\mathbb{R}^{N}\right), v(x)=u(x), \text { for }|x-\beta(u)| \geq R, \\
& \left.\|v\|_{H^{1}(|x-\beta(u)| \geq R)}<\nu\right\}(R \gg 1)
\end{array}
$$


and it is defined the tail minimizing operator as a unique minimizer of (1.10), which helps to control the behavior of solutions outside small balls. Indeed, to justify such approaches, in [9, 15, 16] it is necessary to show that solutions of penalized problems are small in the penalized region and this fact relies on the decay property of linear equations outside small balls. To this aim it is crucial the exponential decay of solutions, which are valid if $f(s)=|s|^{p-2} s$, $p \in\left[2, \frac{N+\alpha}{N-2}\right)$ or (1.9) holds. Of course, for the argument in [10, 11], solvability and uniqueness of solutions of the minimizing problem (1.10) is required.

Moreover we note that $J_{\varepsilon}(u)$ defined in (1.6) is well-defined and of class $C^{1}$ on $H^{1}\left(\mathbb{R}^{N}\right)$ under the conditions (f1)-(f5), especially for $f(s)=|s|^{p-2} s$, $p \in\left(\frac{N+\alpha}{N}, \frac{N+\alpha}{N-2}\right)$. However for a given $u \in H^{1}\left(\mathbb{R}^{N}\right)$, the minimizing problem (1.10) (or corresponding linear problem) outside small balls is not solvable due to non-local feature of the problem under (f1)-(f5) and in [38, 48], the authors assume $f(s)=|s|^{p-2} s, p \in\left[2, \frac{N+\alpha}{N-2}\right)$ or (1.9). Actually in [38], cases $p \in\left(2, \frac{N+\alpha}{N-2}\right)$ and $p=2$ are discussed separately. See [38], especially Section 6.1 .

In this paper we take a new approach, which is inspired by [10, 11], and we find an operator $\tau_{\varepsilon}$, which has similar properties to the tail minimizing operator in [10, 11], via a deformation argument. Our operator $\tau_{\varepsilon}: N_{\varepsilon}(\nu) \rightarrow$ $N_{\varepsilon}(5 \nu)$ has the following properties:

$$
\begin{aligned}
& \tau_{\varepsilon}(u)(x)=u(x) \quad \text { for }|x-\beta(u)| \leq \frac{1}{\sqrt{\varepsilon}}, \\
& J_{\varepsilon}\left(\tau_{\varepsilon}(u)\right) \leq J_{\varepsilon}(u), \\
& \left\|\tau_{\varepsilon}(u)\right\|_{H^{1}\left(|x-\beta(u)| \geq \frac{2}{\sqrt{\varepsilon}}\right)} \leq \widetilde{\rho}_{\varepsilon},
\end{aligned}
$$

where $\widetilde{\rho}_{\varepsilon}$ is independent of $u \in N_{\varepsilon}(\nu)$ and $\widetilde{\rho}_{\varepsilon} \rightarrow 0$ as $\varepsilon \rightarrow 0$.

Using the operator $\tau_{\varepsilon}$ together with the standard deformation argument, we can directly deal with $J_{\varepsilon}(u)$ in $N_{\varepsilon}(\nu)$. Virtue of our approach is the following; first we can find a critical point without introducing penalization, which simplifies the argument, second, we argue without linear equations outside small balls nor the uniform exponential decay of solutions, so we are able to deal with locally sublinear case, for example, $f(s)=|s|^{p-2} s$, $p \in\left(\frac{N+\alpha}{2}, 2\right)$. We believe that our new approach is applicable to a wide class of singular perturbation problems.

We also study a multiplicity of positive single-peak solutions of (1.1) under (f1)-(f5), relating the number of positive solutions to the topology of the critical set $K$ of $V(x)$ in the spirit of well-known results Bahri, Coron 
[4] and Benci, Cerami [6, 7, 8] for semilinear elliptic problems with Dirichlet boundary condition.

Analysis of the topological changes between two level sets of $J_{\varepsilon}(u)$ in a small neighborhood $N_{\varepsilon}(\nu)$ of expected solutions is essential in our argument. This approach is taken for nonlinear Schrödinger equation and nonlinear magnetic Schrödinger equations in [15, 16]. We extend these ideas to nonlocal setting. We note that we do not require any monotonicity assumption on $f$. As a consequence we can not use advantage of Nehari manifolds and we use relative category and cup-length to estimate the topological changes.

Our result is

Theorem 1.4 Suppose $N \geq 3$ and that (f1)-(f5) and (V1)-(V2) hold. Then, for sufficiently small $\varepsilon>0$, (1.1) has at least $\operatorname{cupl}(K)+1$ positive solutions $v_{\varepsilon}^{i}$, $i=1, \ldots, \operatorname{cupl}(K)+1$ concentrating as $\varepsilon \rightarrow 0$ in $K$, where $\operatorname{cupl}(K)$ denotes the cup-length defined with Alexander-Spanier cohomology with coefficients in the field $\mathbb{F}$.

Remark 1.5 If $K=\mathbb{S}^{N-1}$, the $N-1$ dimensional sphere in $\mathbb{R}^{N}$, then $\operatorname{cupl}(K)+1=\operatorname{cat}(K)=2$. If $K=\mathbb{T}^{N}$ is the $N$-dimensional torus, then $\operatorname{cupl}(K)+1=\operatorname{cat}(K)=N+1$. However in general $\operatorname{cupl}(K)+1 \leq \operatorname{cat}(K)$.

Remark 1.6 When we say that the solutions $v_{\varepsilon}^{i}, i=1, \ldots, \operatorname{cupl}(K)+1$ of Theorem 1.4 ( $v_{\varepsilon}$ of Theorem 1.1 resp.) concentrate when $\varepsilon \rightarrow 0$ in $K$, we mean that there exists a maximum point $x_{\varepsilon}^{i}$ of $v_{\varepsilon}^{i}$ ( $x_{\varepsilon}$ of $v_{\varepsilon}$ resp.) such that $\lim _{\varepsilon \rightarrow 0} \operatorname{dist}\left(x_{\varepsilon}^{i}, K\right)=0\left(\lim _{\varepsilon \rightarrow 0} \operatorname{dist}\left(x_{\varepsilon}, K\right)=0\right.$ resp.) and that for any such $x_{\varepsilon}^{i}, w_{\varepsilon}^{i}(x)=v_{\varepsilon}^{i}\left(\varepsilon\left(x+x_{\varepsilon}^{i}\right)\right)\left(w_{\varepsilon}(x)=v_{\varepsilon}\left(\varepsilon\left(x+x_{\varepsilon}\right)\right)\right.$ resp.) converges, up to a subsequence, uniformly to a least energy solution $U$ of (1.8).

Since Theorem 1.1 can be regarded as a special case of Theorem 1.4, we mainly deal with Theorem 1.4. To study our non-local problem, we develop several new tools.

This paper is organized as follows: In Section 2, we develop useful estimates for a non-local term $\int_{\mathbb{R}^{N}}\left(I_{\alpha} * F(u)\right) F(u) d x$, which we use throughout this paper. In Section 3, we study the limit problem (1.8). As a typical feature of (1.8), the problem has 3 different types of homogeneities and we employ new ideas as a new Pohozaev type function $\Lambda(u)$. This method can be useful to study other elliptic problem involving different types of scaling. We also introduce a center of mass in $N_{\varepsilon}(\nu)$. In Section 4, we give a gradient 
estimate of $J_{\varepsilon}(u)$ in a neighborhood of $\partial N_{\varepsilon}(\nu)$, which will play an essential role in the proof. In Section 5, we develop a deformation argument for a tail of $u \in N_{\varepsilon}(\nu)$, i.e., outside small balls, to construct a map $\tau_{\varepsilon}$. In Sections 6 [7, using ideas from [15, 16], we analyze change of the topology of level sets of $J_{\varepsilon}(u)$ in $N_{\varepsilon}(\nu)$ via relative cup-length and give a proof of Theorem 1.4.

For Theorem 1.1, we can give a simpler proof without using cup-length nor category, which we believe of interest itself. We give an outline of the simpler proof in Section 8, in which we use a localized mountain pass argument.

\section{Preliminaries}

In what follows we use the notation:

$$
\begin{aligned}
& \|u\|_{H^{1}}=\left(\int_{\mathbb{R}^{N}}|\nabla u|^{2}+u^{2} d x\right)^{1 / 2}, \\
& \|u\|_{r}=\left(\int_{\mathbb{R}^{N}}|u|^{r} d x\right)^{1 / r} \text { for } r \in[1, \infty), \\
& B(p, R)=\left\{x \in \mathbb{R}^{N} ;|x-p|<R\right\}, \\
& B(p, R)^{c}=\mathbb{R}^{N} \backslash B(p, R) \text { for } p \in \mathbb{R}^{N} \text { and } R>0 .
\end{aligned}
$$

We study the multiplicity of solutions to (1.5) via a variational method. That is, we look for critical points of the functional $J_{\varepsilon} \in C^{1}\left(H^{1}\left(\mathbb{R}^{N}\right), \mathbb{R}\right)$ defined by

$$
J_{\varepsilon}(u)=\frac{1}{2}\|\nabla u\|_{2}^{2}+\frac{1}{2} \int_{\mathbb{R}^{N}} V(\varepsilon x) u^{2} d x-\frac{1}{2} \mathcal{D}(u)
$$

where $\mathcal{D}(u)=\int_{\mathbb{R}^{N}}\left(I_{\alpha} * F(u)\right) F(u) d x$. The critical points of $J_{\varepsilon}$ are clearly solutions of (1.5).

Proposition 2.1 Let $p, r>1$ and $\alpha \in(0, N)$ with $\frac{1}{p}+\frac{1}{r}=\frac{N+\alpha}{N}$. Then there exists a constant $C=C(N, \alpha, p, r)>0$ such that

$$
\left|\int_{\mathbb{R}^{N}}\left(I_{\alpha} * f\right) g d x\right| \leq C\|f\|_{p}\|g\|_{r}
$$

for all $f \in L^{p}\left(\mathbb{R}^{N}\right)$ and $g \in L^{r}\left(\mathbb{R}^{N}\right)$. 
Proposition 2.1 can be derived from the following generalized HardyLittlewood-Sobolev inequality. In the following proposition, $L_{w}^{q}\left(\mathbb{R}^{N}\right)$ is the weak $L^{q}$ space. See [31, 32] for the definition. We denote by $\|\cdot\|_{q, w}$ the usual $\operatorname{norm}$ in $L_{w}^{q}\left(\mathbb{R}^{N}\right)$.

Proposition 2.2 Let $p, q, r \in(1, \infty)$ satisfies $\frac{1}{p}+\frac{1}{q}+\frac{1}{r}=2$. Then there exists a constant $N_{p, q, r}>0$ such that for any $f \in L^{p}\left(\mathbb{R}^{N}\right), g \in L^{r}\left(\mathbb{R}^{N}\right)$ and $h \in L_{w}^{q}\left(\mathbb{R}^{N}\right)$

$$
\left|\int_{\mathbb{R}^{N} \times \mathbb{R}^{N}} f(x) h(x-y) g(y) d x d y\right| \leq N_{p, q, r}\|f\|_{p}\|g\|_{r}\|h\|_{q, w} .
$$

In what follows, we denote various constants, which are independent of $u \in H^{1}\left(\mathbb{R}^{N}\right)$, by $C, C^{\prime}, C^{\prime \prime}, \cdots$.

By Proposition 2.1, we have for $u, v \in H^{1}\left(\mathbb{R}^{N}\right)$

$$
\begin{aligned}
|\mathcal{D}(u)| & \leq C\|F(u)\|_{\frac{2 N}{N+\alpha}}^{2} \leq C\left(\|u\|_{2}^{2}+\|u\|_{\frac{2 N}{N-2}}^{\frac{2 N}{N-2}}\right)^{\frac{N+\alpha}{N}}, \\
\left|\mathcal{D}^{\prime}(u) u\right| & \leq C\|F(u)\|_{\frac{2 N}{N+\alpha}}\|f(u) u\|_{\frac{2 N}{N+\alpha}} \leq C^{\prime}\left(\|u\|_{2}^{2}+\|u\|_{\frac{2 N}{N-2}}^{\frac{2 N}{N-2}}\right)^{\frac{N+\alpha}{N}} \\
\left|\mathcal{D}^{\prime}(u) v\right| & \leq C\|F(u)\|_{\frac{2 N}{N+\alpha}}\|f(u) v\|_{\frac{2 N}{N+\alpha}}^{N+\alpha} \\
& \leq C^{\prime}\left(\|u\|_{2}^{2}+\|u\|_{\frac{2 N}{N-2}}^{\frac{2 N}{N-2}}\right)^{\frac{N+\alpha}{2 N}}\left(\|u\|_{2}^{\frac{2 \alpha}{N+\alpha}}+\|u\|_{\frac{2 N}{N-2}}^{\frac{2 N}{N-2} \frac{2+\alpha}{N+\alpha}}\right)^{\frac{N+\alpha}{2 N}}\|v\|_{H^{1}} .
\end{aligned}
$$

We also have

$$
\begin{aligned}
J_{\varepsilon}(u) & \geq \frac{1}{2}\|\nabla u\|_{2}^{2}+\frac{V}{2}\|u\|_{2}^{2}-C\|F(u)\|_{\frac{2 N}{N+\alpha}}^{2}, \\
J_{\varepsilon}^{\prime}(u) u & \geq\|\nabla u\|_{2}^{2}+\underline{V}\|u\|_{2}^{2}-C\|F(u)\|_{\frac{2 N}{N+\alpha}}\|f(u) u\|_{\frac{2 N}{N+\alpha}} .
\end{aligned}
$$

In particular, $J_{\varepsilon}(u)$ has mountain pass geometry uniformly in $\varepsilon \in(0,1]$ and we have

Corollary 2.3 There exist $\nu_{0}>0$ and $c_{0}>0$ such that

$$
J_{\varepsilon}(u) \geq c_{0}\|u\|_{H^{1}}^{2}, \quad J_{\varepsilon}^{\prime}(u) u \geq c_{0}\|u\|_{H^{1}}^{2}
$$

for all $u \in H^{1}\left(\mathbb{R}^{N}\right)$ with $\|u\|_{H^{1}} \leq \nu_{0}$. 
For latter uses, we give

Lemma 2.4 Let $p, r>1$ and $\alpha \in(0, N)$ with $\frac{1}{p}+\frac{1}{r}<\frac{N+\alpha}{N}$. Then there exists a constant $D_{R}>0$ depending on $R>0$ such that

$$
D_{R} \rightarrow 0 \quad \text { as } R \rightarrow \infty
$$

and

$$
\left|\int_{\mathbb{R}^{N}}\left(I_{\alpha} * f\right) g d x\right| \leq D_{R}\|f\|_{p}\|g\|_{r}
$$

for all $f \in L^{p}\left(\mathbb{R}^{N}\right)$ and $g \in L^{r}\left(\mathbb{R}^{N}\right)$ with $\operatorname{dist}(\operatorname{supp} f$, supp $g) \geq R$.

Proof. We set

$$
I_{\alpha}^{R}(x)= \begin{cases}\frac{1}{|x|^{N-\alpha}} & \text { if }|x| \geq R \\ 0 & \text { if }|x|<R\end{cases}
$$

We note that $\left\|I_{\alpha}^{R}\right\|_{\frac{N}{N-\alpha}, w}$ remains bounded as $R \rightarrow \infty$ but $\left\|I_{\alpha}^{R}\right\|_{\frac{N}{N-\alpha}, w} \not \rightarrow 0$ as $R \rightarrow \infty$, while $\left\|I_{\alpha}^{R}\right\|_{q} \rightarrow 0$ as $R \rightarrow \infty$ for any $q \in\left(\frac{N}{N-\alpha}, \infty\right)$.

For $\frac{1}{p}+\frac{1}{r}<\frac{N+\alpha}{N}$, we find $q \in\left(\frac{N}{N-\alpha}, \infty\right)$ such that $\frac{1}{p}+\frac{1}{q}+\frac{1}{r}=2$. For $f \in L^{p}\left(\mathbb{R}^{N}\right)$ and $g \in L^{r}\left(\mathbb{R}^{N}\right)$ with $\operatorname{dist}(\operatorname{supp} f, \operatorname{supp} g) \geq R$, the HausdorffYoung inequality implies

$$
\left|\int_{\mathbb{R}^{N}}\left(I_{\alpha} * f\right) g d x\right|=\left|\int_{\mathbb{R}^{N}}\left(I_{\alpha}^{R} * f\right) g d x\right| \leq\left\|I_{\alpha}^{R}\right\|_{q}\|f\|_{p}\|g\|_{r} .
$$

Setting $D_{R}=\left\|I_{\alpha}^{R}\right\|_{q}$, we have the conclusion.

As a corollary to Proposition 2.1 and Lemma 2.4, we have

Corollary 2.5 Suppose that $F(s): \mathbb{R} \rightarrow \mathbb{R}$ satisfy (f1)-(f3). Then there exists a constant $m_{R}>0$ such that

$$
m_{R} \rightarrow 0 \quad \text { as } R \rightarrow \infty
$$

and for any $u \in H^{1}\left(\mathbb{R}^{N}\right)$ and $g \in L^{\frac{2 N}{N+\alpha}}\left(\mathbb{R}^{N}\right)$ with $\operatorname{dist}(\operatorname{supp} u$, supp $g) \geq R$

$$
\left|\int_{\mathbb{R}^{N}}\left(I_{\alpha} * F(u)\right) g d x\right| \leq m_{R} \sigma\left(\|u\|_{H^{1}}\right)^{\frac{N+\alpha}{2 N}}\|g\|_{\frac{2 N}{N+\alpha}},
$$

where

$$
\sigma(t)=t^{2}+t^{\frac{2 N}{N-2}}
$$


Proof. We fix $q \in\left(2, \frac{2 N}{N-2}\right)$ and write $q=\frac{2 N}{N-2} \theta$ with $\theta \in\left(\frac{N-2}{N}, 1\right)$. By (f3), for any $\delta>0$ there exists $C_{\delta}>0$ such that

$$
|F(s)| \leq \delta \sigma(s)^{\frac{N+\alpha}{2 N}}+C_{\delta}|s|^{q \frac{N+\alpha}{2 N}} \quad \text { for all } s \in \mathbb{R}
$$

Setting

$$
\begin{aligned}
& H_{1}(s)= \begin{cases}\delta \sigma(s)^{\frac{N+\alpha}{2 N}} & \text { if }|F(s)| \geq \delta \sigma(s)^{\frac{N+\alpha}{2 N}} \\
|F(s)| & \text { if }|F(s)|<\delta \sigma(s)^{\frac{N+\alpha}{2 N}}\end{cases} \\
& H_{2}(s)=|F(s)|-H_{1}(s)
\end{aligned}
$$

we have

$$
\begin{aligned}
|F(s)| & =H_{1}(s)+H_{2}(s) \\
H_{1}(s) & \leq \delta \sigma(s)^{\frac{N+\alpha}{2 N}} \\
H_{2}(s) & \leq C_{\delta}|s|^{q \frac{N+\alpha}{2 N}}
\end{aligned}
$$

We note for $u \in H^{1}\left(\mathbb{R}^{N}\right)$

$$
\begin{aligned}
\left\|H_{1}(u)\right\|_{\frac{2 N}{N+\alpha}} & \leq \delta\left(\|u\|_{2}^{2}+\|u\|_{\frac{2 N}{N-2}}^{\frac{2 N}{N-2}}\right)^{\frac{N+\alpha}{2 N}} \leq \delta C^{\prime} \sigma\left(\|u\|_{H^{1}}\right)^{\frac{N+\alpha}{2 N}} \\
\left\|H_{2}(u)\right\|_{\frac{2 N}{N+\alpha} \frac{1}{\theta}} & \leq C_{\delta}\|u\|_{q / \theta}^{q \frac{N+\alpha}{2 N}}=C_{\delta}\|u\|_{\frac{2 N}{N-2}}^{q \frac{N+\alpha}{2 N}} \leq C_{\delta}^{\prime}\|u\|_{H^{1}}^{q \frac{N+\alpha}{2 N}} \\
& \leq C_{\delta}^{\prime \prime} \sigma\left(\|u\|_{H^{1}}\right)^{\frac{N+\alpha}{2 N}}
\end{aligned}
$$

Thus,

$$
\begin{aligned}
\left|\int_{\mathbb{R}^{N}}\left(I_{\alpha} * F(u)\right) g d x\right| & \leq \int_{\mathbb{R}^{N}}\left(I_{\alpha} * H_{1}(u)\right)|g| d x+\int_{\mathbb{R}^{N}}\left(I_{\alpha} * H_{2}(u)\right)|g| d x \\
& \leq C\left\|H_{1}(u)\right\|_{\frac{2 N}{N+\alpha}}\|g\|_{\frac{2 N}{N+\alpha}}+D_{R}\left\|H_{2}(u)\right\|_{\frac{2 N}{N+\alpha} \frac{1}{\theta}}\|g\|_{\frac{2 N}{N+\alpha}} \\
& \leq\left(C C^{\prime} \delta+D_{R} C_{\delta}^{\prime \prime}\right) \sigma\left(\|u\|_{H^{1}}\right)^{\frac{N+\alpha}{2 N}}\|g\|_{\frac{2 N}{N+\alpha}}
\end{aligned}
$$

We set

$$
\begin{aligned}
m_{R} & \equiv \sup _{u \in H^{1}\left(\mathbb{R}^{N}\right) \backslash\{0\}} \frac{\left|\int_{\mathbb{R}^{N}}\left(I_{\alpha} * F(u)\right) g d x\right|}{\sigma\left(\|u\|_{H^{1}}\right)^{\frac{N+\alpha}{2 N}}\|g\|_{\frac{2 N}{N+\alpha}}} \\
& \leq C C^{\prime} \delta+D_{R} C_{\delta}^{\prime \prime} .
\end{aligned}
$$


It follows from Lemma 2.4 that

$$
\limsup _{R \rightarrow \infty} m_{R} \leq C C^{\prime} \delta
$$

Since $\delta>0$ is arbitrary, we have $m_{R} \rightarrow 0$ as $R \rightarrow \infty$.

For $R>0$ we choose functions $\zeta_{R}(s), \widetilde{\zeta}_{R}(s) \in C^{\infty}(\mathbb{R}, \mathbb{R})$ such that

$$
\zeta_{R}(s)=\left\{\begin{array}{ll}
1 & \text { for } s \leq R, \\
0 & \text { for } s \geq R+2,
\end{array} \quad \widetilde{\zeta}_{R}(s)= \begin{cases}0 & \text { for } s \leq R-2 \\
1 & \text { for } s \geq R\end{cases}\right.
$$

and $\zeta_{R}(s), \widetilde{\zeta}_{R}(s) \in[0,1],-\zeta_{R}^{\prime}(s), \widetilde{\zeta}_{R}^{\prime}(s) \in[0,1]$ for all $s \in \mathbb{R}$. We also set

$$
\chi_{R}(s)=\left\{\begin{array}{ll}
1 & \text { for } s \leq R, \\
0 & \text { for } s>R
\end{array}, \quad \tilde{\chi}_{R}(s)=1-\chi_{R}(s) .\right.
$$

Corollary 2.6 For a fixed $M>0$, suppose that $u, v \in H^{1}\left(\mathbb{R}^{N}\right)$ satisfy

$$
\|u\|_{H^{1}},\|v\|_{H^{1}} \leq M
$$

Then there exist $C_{1}, C_{2}>0$ such that for any $p \in \mathbb{R}^{N}$ and $R, L>4$ we have

(i) $\left|\mathcal{D}(u)-\mathcal{D}\left(\zeta_{R}(|x-p|) u\right)-\mathcal{D}\left(\widetilde{\zeta}_{R+L}(|x-p|) u\right)\right| \leq C_{1}\|u\|_{H^{1}(|x-p| \in[R, R+L])}^{\frac{N+\alpha}{N}}+$ $\mathrm{C}_{2} m_{L}$.

(ii) $\mid \mathcal{D}^{\prime}(u) v-\mathcal{D}^{\prime}\left(\zeta_{R}(|x-p|) u\right)\left(\zeta_{R}(|x-p|) v\right)$

$-\mathcal{D}^{\prime}\left(\widetilde{\zeta}_{R+L}(|x-p|) u\right)\left(\widetilde{\zeta}_{R+L}(|x-p|) v\right) \mid$

$\leq C_{1} \max \left\{\|u\|_{H^{1}(|x-p| \in[R, R+L])},\|v\|_{H^{1}(|x-p| \in[R, R+L])}\right\}^{\frac{N+\alpha}{N}}+C_{2} m_{L}$.

(iii) $\left|\left(\mathcal{D}^{\prime}(u)-\mathcal{D}^{\prime}\left(\widetilde{\zeta}_{R+L}(|x-p|) u\right)\right)\left(\widetilde{\zeta}_{R+L}(|x-p|) v\right)\right|$

$\leq C_{1} \max \left\{\|u\|_{H^{1}(|x-p| \in[R, R+L])},\|v\|_{H^{1}(|x-p| \in[R, R+L])}\right\}^{\frac{N+\alpha}{N}}+C_{2} m_{L}$.

Proof. We may assume $p=0$ and we prove just (ii). (i) and (iii) can be shown in a similar way.

We note that

$$
\mathcal{D}^{\prime}(u) v=\int_{\mathbb{R}^{N}}\left(I_{\alpha} * F(u)\right) f(u) v d x .
$$


We set

$$
\begin{aligned}
& F_{A}=F\left(\chi_{R}(|x|) u\right), \quad F_{B}=F\left(\widetilde{\chi}_{R+L}(|x|) u\right), \\
& F_{C}=F\left(\left(1-\chi_{R}(|x|)-\widetilde{\chi}_{R+L}(|x|)\right) u\right), \\
& g_{A}=f\left(\chi_{R}(|x|) u\right) \chi_{R}(|x|) v, \quad g_{B}=f\left(\widetilde{\chi}_{R+L}(|x|) u\right) \widetilde{\chi}_{R+L} v(|x|), \\
& g_{C}=f\left(\left(1-\chi_{R}(|x|)-\widetilde{\chi}_{R+L}(|x|)\right) u\right)\left(\left(1-\chi_{R}(|x|)-\widetilde{\chi}_{R+L}(|x|)\right) v\right) .
\end{aligned}
$$

We also set $\delta=\max \left\{\|u\|_{H^{1}(|x| \in[R, R+L])},\|v\|_{H^{1}(|x| \in[R, R+L])}\right\}$.

First we show for some constants $C_{1}, C_{2}>0$ independent of $u, v$

$$
\begin{aligned}
& \left.\mid \int_{\mathbb{R}^{N}}\left(I_{\alpha} * F(u)\right) f(u) v d x-\int_{\mathbb{R}^{N}}\left(I_{\alpha} * F_{A}\right) g_{A} d x-\int_{\mathbb{R}^{N}}\left(I_{\alpha} * F_{B}\right)\right) g_{B} d x \mid \\
\leq & C_{1} \delta^{\frac{N+\alpha}{N}}+C_{2} m_{L} .
\end{aligned}
$$

In fact,

$$
\begin{aligned}
& \int_{\mathbb{R}^{N}}\left(I_{\alpha} * F(u)\right) v d x \\
= & \int_{\mathbb{R}^{N}}\left(I_{\alpha} *\left(F_{A}+F_{B}+F_{C}\right)\right)\left(g_{A}+g_{B}+g_{C}\right) d x \\
= & \int_{\mathbb{R}^{N}}\left(I_{\alpha} * F_{A}\right) g_{A} d x+\int_{\mathbb{R}^{N}}\left(I_{\alpha} * F_{B}\right) g_{B} d x \\
& +\int_{\mathbb{R}^{N}}\left(I_{\alpha} * F_{A}\right) g_{B} d x+\int_{\mathbb{R}^{N}}\left(I_{\alpha} * F_{B}\right) g_{A} d x \\
& +\int_{\mathbb{R}^{N}}\left(I_{\alpha} * F_{C}\right)\left(g_{A}+g_{B}\right) d x+\int_{\mathbb{R}^{N}}\left(I_{\alpha} *\left(F_{A}+F_{B}\right)\right) g_{C} d x \\
& +\int_{\mathbb{R}^{N}}\left(I_{\alpha} * F_{C}\right) g_{C} d x
\end{aligned}
$$

By Corollary 2.5,

$$
\left|\int_{\mathbb{R}^{N}}\left(I_{\alpha} * F_{A}\right) g_{B} d x+\int_{\mathbb{R}^{N}}\left(I_{\alpha} * F_{B}\right) g_{A} d x\right| \leq C m_{L} .
$$

We can easily see that for some constant $C$ depending only on $M$

$$
\begin{aligned}
& \left\|F_{A}\right\|_{\frac{2 N}{N+\alpha}},\left\|F_{B}\right\|_{\frac{2 N}{N+\alpha}},\left\|g_{A}\right\|_{\frac{2 N}{N+\alpha}},\left\|g_{B}\right\|_{\frac{2 N}{N+\alpha}} \leq C, \\
& \left\|F_{C}\right\|_{\frac{2 N}{N+\alpha}},\left\|g_{C}\right\|_{\frac{2 N}{N+\alpha}} \leq C \delta^{\frac{N+\alpha}{N}} .
\end{aligned}
$$


Thus we get (2.5).

Since

$$
\begin{aligned}
& \left\|F_{A}-F\left(\zeta_{R} u\right)\right\|_{\frac{2 N}{N+\alpha}},\left\|F_{B}-F\left(\widetilde{\zeta}_{R+L} u\right)\right\|_{\frac{2 N}{N+\alpha}}, \\
& \left\|g_{A}-f\left(\zeta_{R} u\right)\left(\zeta_{R} v\right)\right\|_{\frac{2 N}{N+\alpha}},\left\|g_{B}-f\left(\widetilde{\zeta}_{R+L} u\right)\left(\widetilde{\zeta}_{R+L} v\right)\right\|_{\frac{2 N}{N+\alpha}} \leq C \delta^{\frac{N+\alpha}{\alpha}},
\end{aligned}
$$

we have the conclusion (ii).

We will use Corollary $\left[2.6\right.$ with $M=\sup _{\omega \in S_{m_{0}}, s \in\left[\frac{1}{2}, \frac{3}{2}\right]}\|\omega(x / s)\|_{H^{1}}+1$ repeatedly.

\section{Limit problem}

\subsection{Limit problem}

Let $m_{0}, K$ be introduced in (V2). For $d>0$, let $K_{d}=\left\{x \in \mathbb{R}^{N} ; \inf _{y \in K} \mid x-\right.$ $y \mid<d\}$ be a $d$-neighborhood of $K$.

In what follows we denote $K=\left\{x \in \Omega ; V(x)=m_{0}\right\}$ where $m_{0}$ is introduced in (V1) and $K_{d}$ a $d$-neighborhood of $K$.

We can choose $d_{0}>0$ small such that $K_{2 d_{0}} \subset \Omega$ and

$$
V(x) \geq m_{0}+\rho_{0} \quad \text { for all } x \in K_{2 d_{0}} \backslash K_{\frac{1}{2} d_{0}}
$$

for some $\rho_{0}>0$ and the conclusion of Lemma 7.5 in the following Section 7 holds for $d=d_{0}$.

For $a>0$ we define a functional $L_{a} \in C^{1}\left(H^{1}\left(\mathbb{R}^{N}\right), \mathbb{R}\right)$ by

$$
L_{a}(u)=\frac{1}{2}\|\nabla u\|_{2}^{2}+\frac{a}{2}\|u\|_{2}^{2}-\frac{1}{2} \mathcal{D}(u) .
$$

Especially $L_{m_{0}}(u)$ is associated to the limit problem

$$
-\Delta u+m_{0} u=\left(I_{\alpha} * F(u)\right) f(u), \quad u \in H^{1}\left(\mathbb{R}^{N}\right) .
$$

We denote by $E\left(m_{0}\right)$ the least energy level for (3.2). That is,

$$
E\left(m_{0}\right)=\inf \left\{L_{m_{0}}(u) ; u \neq 0, L_{m_{0}}^{\prime}(u)=0\right\} .
$$

In [37] it is proved that there exists a least energy solution of (3.2) if (f1)(f4) are satisfied. They proved that if $f$ is odd and has a constant sign on 
$(0,+\infty)$, then every ground state solution of (3.2) has constant sign and is radially symmetric with respect to some point in $\mathbb{R}^{N}$.

Also it is showed that each solution of (3.2) satisfies the Pohozaev's identity

$$
\frac{N-2}{2}\|\nabla u\|_{2}^{2}+\frac{N}{2} m_{0}\|u\|_{2}^{2}=\frac{N+\alpha}{2} \mathcal{D}(u)
$$

We set

$S_{m_{0}}=\left\{\omega \in H^{1}\left(\mathbb{R}^{N}\right) \backslash\{0\} ; L_{m_{0}}^{\prime}(\omega)=0, L_{m_{0}}(\omega)=E\left(m_{0}\right), \omega(0)=\max _{x \in \mathbb{R}^{N}} \omega(x)\right\}$

Arguing as in [37] we can prove that $S_{m_{0}}$ is compact in $H^{1}\left(\mathbb{R}^{N}\right)$ and that its elements have a uniform decay. Especially,

$$
\|\omega\|_{H^{1}\left(B(0, R)^{c}\right)} \rightarrow 0 \text { as } R \rightarrow \infty \text { uniformly in } \omega \in S_{m_{0}} .
$$

Moreover, they have a uniform exponential decay if $\lim _{\sup } \rightarrow 0 \frac{f(x)}{s}<\infty$ and they have a uniform polynomial decay if $F(s)=|s|^{p}$ with $p \in\left(\frac{\stackrel{N}{N}+\alpha}{N}, 2\right)$. See Moroz-Van Schaftingen [36].

\subsection{A Pohozaev type function}

To study the scaling property of the limit equation (3.2), we define a functional

$$
J(\lambda, u)=L_{m_{0}}(u(\cdot / \lambda))=\frac{\lambda^{N-2}}{2}\|\nabla u\|_{2}^{2}+m_{0} \frac{\lambda^{N}}{2}\|u\|_{2}^{2}-\frac{\lambda^{N+\alpha}}{2} \mathcal{D}(u) .
$$

For any $u \in H^{1}\left(\mathbb{R}^{N}\right) \backslash\{0\}$, we have

$$
\frac{\partial}{\partial \lambda} J(\lambda, u)=\frac{(N-2) \lambda^{N-3}}{2}\|\nabla u\|_{2}^{2}+m_{0} \frac{N \lambda^{N-1}}{2}\|u\|_{2}^{2}-\frac{(N+\alpha) \lambda^{N+\alpha-1}}{2} \mathcal{D}(u) .
$$

Since $\lim _{\lambda \rightarrow 0^{+}} \frac{1}{\lambda^{N-2}} J(\lambda, u)>0, \lim _{\lambda \rightarrow \infty} J(\lambda, u)=-\infty$ and moreover for any critical point $\lambda \in(0, \infty)$ of $\lambda \mapsto J(\lambda, u)$,

$$
\frac{\partial^{2}}{\partial \lambda^{2}} J(\lambda, u)=-\left\{\frac{(\alpha+2)(N-2)}{2} \lambda^{N-4}\|\nabla u\|_{2}^{2}+\frac{\alpha N m_{0}}{2} \lambda^{N-2}\|u\|_{2}^{2}\right\}<0
$$

we observe that for any $u \in H^{1}\left(\mathbb{R}^{N}\right) \backslash\{0\},(0, \infty) \rightarrow \mathbb{R} ; \lambda \mapsto J(\lambda, u)$ has a unique critical point $\Lambda(u)$, which is a non-degenerate local maximum. We 
note that $\Lambda(u): H^{1}\left(\mathbb{R}^{N}\right) \backslash\{0\} \rightarrow \mathbb{R}$ is continuous and by the Pohozaev identity,

$$
\Lambda(u)=1 \quad \text { for all } u \in S_{m_{0}}
$$

We also have

$$
\Lambda(u)=s \quad \text { for } u=\omega\left(\frac{x-p}{s}\right) \text { with } \omega \in S_{m_{0}}, s>0, p \in \mathbb{R}^{N} .
$$

Lemma 3.1 There exist $C_{0}>0$ and $s_{0} \in\left(0, \frac{1}{2}\right)$ such that for any $v \in$ $H^{1}\left(\mathbb{R}^{N}\right) \backslash\{0\}$ with $\Lambda(v)=1$ and $s \in\left[1-2 s_{0}, 1+2 s_{0}\right]$

$$
L_{m_{0}}\left(v\left(\frac{x}{s}\right)\right) \geq E\left(m_{0}\right)\left(1-C_{0}(s-1)^{2}\right) .
$$

Proof. As in [37] (see also [28]), we can prove the following characterization:

$$
E\left(m_{0}\right)=\inf \left\{L_{m_{0}}(u) ; u \neq 0, \Lambda(u)=1\right\} .
$$

For $v \in H^{1}\left(\mathbb{R}^{N}\right) \backslash\{0\}$ with $\Lambda(v)=1$ and $s>0$,

$$
\begin{aligned}
& L_{m_{0}}\left(v\left(\frac{x}{s}\right)\right)=\frac{s^{N-2}}{2}\|\nabla v\|_{2}^{2}+\frac{s^{N}}{2} m_{0}\|v\|_{2}^{2}-\frac{s^{N+\alpha}}{2} \mathcal{D}(v) \\
& \quad=\frac{1}{2}\left(s^{N-2}-\frac{N-2}{N+\alpha} s^{N+\alpha}\right)\|\nabla v\|_{2}^{2}+\frac{1}{2}\left(s^{N}-\frac{N}{N+\alpha} s^{N+\alpha}\right) m_{0}\|v\|_{2}^{2} \\
& \quad \equiv \frac{1}{2} g(s)\|\nabla v\|_{2}^{2}+\frac{1}{2} h(s) m_{0}\|v\|_{2}^{2} .
\end{aligned}
$$

It is easy to see that $g(s)$ and $h(s)$ take their unique maxima at $s=1$ and $g^{\prime \prime}(1)<0, h^{\prime \prime}(1)<0$. Thus there exists $C_{0}>0$ and $s_{0} \in\left(0, \frac{1}{2}\right)$ such that

$$
\begin{aligned}
& g(s) \geq g(1)\left(1-C_{0}(s-1)^{2}\right), \\
& h(s) \geq h(1)\left(1-C_{0}(s-1)^{2}\right)
\end{aligned}
$$

for all $s \in\left[1-2 s_{0}, 1+2 s_{0}\right]$. Therefore we have

$$
\begin{aligned}
L_{m_{0}}\left(v\left(\frac{x}{s}\right)\right) & \geq\left(\frac{1}{2} g(1)\|\nabla v\|_{2}^{2}+\frac{1}{2} h(1) m_{0}\|v\|_{2}^{2}\right)\left(1-C_{0}(s-1)^{2}\right) \\
& =L_{m_{0}}(v)\left(1-C_{0}(s-1)^{2}\right) .
\end{aligned}
$$

By (3.7), we have the conclusion of Lemma 3.1. 
Corollary 3.2 Assume that $u \in H^{1}\left(\mathbb{R}^{N}\right) \backslash\{0\}$ satisfies $\Lambda(u) \in\left[1-2 s_{0}, 1+\right.$ $\left.2 s_{0}\right]$. Then

$$
L_{m_{0}}(u) \geq E\left(m_{0}\right)\left(1-C_{0}(\Lambda(u)-1)^{2}\right) \text {. }
$$

Proof. Set $v(x)=u(\Lambda(u) x)$. Then we have $\Lambda(v)=1$ and $v(x)=v\left(\frac{x}{\Lambda(u)}\right)$. Thus Corollary 3.2 follows from Lemma 3.1 .

Corollary 3.3 Choosing $s_{0}$ smaller, there exists $\delta_{0}>0$ such that for all $\omega \in S_{m_{0}}$ and $s \in\left[1-2 s_{0}, 1+2 s_{0}\right]$

$$
L_{m_{0}+\rho_{0}}\left(\omega\left(\frac{x}{s}\right)\right) \geq E\left(m_{0}\right)+3 \delta_{0} .
$$

Proof. Let $s_{0} \in\left(0, \frac{1}{2}\right)$ be given in Lemma 3.1. For $\omega \in S_{m_{0}}$ and $s \in$ $\left[1-2 s_{0}, 1+2 s_{0}\right]$ we have

$$
\begin{aligned}
& L_{m_{0}+\rho_{0}}\left(\omega\left(\frac{x}{s}\right)\right)=L_{m_{0}}\left(\omega\left(\frac{x}{s}\right)\right)+\frac{1}{2} \rho_{0}\left\|\omega\left(\frac{x}{s}\right)\right\|_{2}^{2} \\
& \geq E\left(m_{0}\right)\left(1-C_{0}(s-1)^{2}\right)+\frac{1}{2} \rho_{0} \min _{\omega \in S_{m_{0}}, s \in\left[1-2 s_{0}, 1+2 s_{0}\right]}\left\|\omega\left(\frac{x}{s}\right)\right\|_{2}^{2} .
\end{aligned}
$$

Setting $\delta_{0}=\frac{1}{6} \rho_{0} \min _{\omega \in S_{m_{0}}, s \in\left[1-2 s_{0}, 1+2 s_{0}\right]}\left\|\omega\left(\frac{x}{s}\right)\right\|_{2}^{2}>0$, and choosing a smaller $s_{0}$, we have (3.8).

In what follows, we fix $s_{0} \in\left(0, \frac{1}{2}\right)$ for which the conclusion of Lemma 3.1 and (3.8) holds.

\subsection{A center of mass}

Let $s_{0} \in\left(0, \frac{1}{2}\right)$ be the number given at the end of the previous section. We set

$$
\widehat{S}=\left\{\omega\left(\frac{x-p}{s}\right) ; \omega \in S_{m_{0}}, p \in \mathbb{R}^{N}, s \in\left[1-s_{0}, 1+s_{0}\right]\right\} .
$$

We also set

$$
\begin{aligned}
\widehat{\rho}(u) & =\inf _{U \in \widehat{S}}\|u-U\|_{H^{1}} \\
& =\inf \left\{\left\|u-\omega\left(\frac{x-p}{s}\right)\right\|_{H^{1}} ; \omega \in S_{m_{0}}, p \in \mathbb{R}^{N}, s \in\left[1-s_{0}, 1+s_{0}\right]\right\} .
\end{aligned}
$$


For $\nu>0$ small we denote $\nu$-neighborhood of $\widehat{S}$ by $\widehat{S}(\nu)$ :

$$
\begin{aligned}
& \widehat{S}(\nu)=\left\{u \in H^{1}\left(\mathbb{R}^{N}\right) ; \widehat{\rho}(u)<\nu\right\} \\
& =\left\{\omega\left(\frac{x-p}{s}\right)+\varphi(x) ; \omega \in S_{m_{0}}, p \in \mathbb{R}^{N}, s \in\left[1-s_{0}, 1+s_{0}\right],\|\varphi\|_{H^{1}}<\nu\right\} .
\end{aligned}
$$

By the compactness of $S_{m_{0}}$ and (3.5), we have

Lemma 3.4 There exists $\nu_{1}>0$ such that for all $u \in \widehat{S}\left(\nu_{1}\right)$

$$
\Lambda(u) \in\left[1-2 s_{0}, 1+2 s_{0}\right] .
$$

Following [10, 11, 15] for $\nu_{2}>0$ small we introduce a center of mass in $\nu_{2}$-neighborhood $\widehat{S}\left(\nu_{2}\right)$ of $\widehat{S}$.

Lemma 3.5 There exist $\nu_{2}>0, R_{0}>0$ and a map $\beta: \widehat{S}\left(\nu_{2}\right) \rightarrow \mathbb{R}^{N}$ such that

$$
|\beta(u)-p| \leq R_{0}
$$

for all $u(x)=\omega\left(\frac{x-p}{s}\right)+\varphi(x) \in \widehat{S}\left(\nu_{2}\right)$ with $p \in \mathbb{R}^{N}, \omega \in S_{m_{0}}, s \in\left[1-s_{0}, 1+s_{0}\right]$, $\|\varphi\|_{H^{1}}<\nu_{2}$. Moreover, $\beta(u)$ has the following properties:

(i) $\beta(u)$ is shift equivariant, that is,

$$
\beta(u(x-y))=\beta(u(x))+y \quad \text { for all } u \in \widehat{S}\left(\nu_{2}\right) \text { and } y \in \mathbb{R}^{N} .
$$

(ii) $\beta(u)$ is locally Lipschitz continuous, that is, there exist constants $c_{1}$, $c_{2}>0$ such that

$$
|\beta(u)-\beta(v)| \leq c_{1}\|u-v\|_{H^{1}} \quad \text { for all } u, v \in \widehat{S}\left(\nu_{2}\right) \text { with }\|u-v\|_{H^{1}} \leq c_{2} .
$$

(iii) $\beta\left(\omega\left(\frac{x-p}{s}\right)\right)=p$ for all $p \in \mathbb{R}^{N}, \omega \in S_{m_{0}}, s \in\left[1-s_{0}, 1+s_{0}\right]$.

The proof is given in [10, 11] in a slightly different situation. We give here a simple proof.

Proof. Set $\widetilde{S}_{m_{0}}=\left\{U(x)=\omega\left(\frac{x}{s}\right) ; \omega \in S_{m_{0}}, s \in\left[1-s_{0}, 1+s_{0}\right]\right\}$. Then we have $\widehat{S}=\left\{U(x-p) ; U \in \widetilde{S}_{m_{0}}, p \in \mathbb{R}^{N}\right\}$. 
Taking into account that $\widetilde{S}_{m_{0}}$ is compact and the uniform decay (3.4), we can define $r_{*}=\min _{U \in \widetilde{S}_{m_{0}}}\|U\|_{H^{1}}>0$ and choose $R_{*}>1$ such that for $U \in \widetilde{S}_{m_{0}}$

$$
\|U\|_{H^{1}\left(|x| \leq R_{*}\right)}>\frac{3}{4} r_{*} \quad \text { and } \quad\|U\|_{H^{1}\left(|x| \geq R_{*}\right)}<\frac{1}{8} r_{*} .
$$

For $u \in H^{1}\left(\mathbb{R}^{N}\right)$ and $p \in \mathbb{R}^{N}$, we define

$$
d(p, u)=\psi\left(\inf _{U \in \widetilde{S}_{m_{0}}}\|u-U(x-p)\|_{H^{1}\left(|x-p| \leq R_{*}\right)}\right),
$$

where $\psi \in C_{0}^{\infty}(\mathbb{R}, \mathbb{R})$ is such that

$$
\begin{aligned}
& \psi(r)= \begin{cases}1 & r \in\left[0, \frac{1}{4} r_{*}\right], \\
0 & r \in\left[\frac{1}{2} r_{*}, \infty\right),\end{cases} \\
& \psi(r) \in[0,1] \quad \text { for all } r \in[0, \infty) .
\end{aligned}
$$

Now let

$$
\beta(u)=\frac{\int_{\mathbb{R}^{N}} q d(q, u) d q}{\int_{\mathbb{R}^{N}} d(q, u) d q} \text { for } u \in \widehat{S}\left(\frac{1}{8} r_{*}\right) .
$$

We shall show that $\beta$ has the desired property.

Let $u \in \widehat{S}\left(\frac{1}{8} r_{*}\right)$ and write $u(x)=U(x-p)+\varphi(x)$ with $U \in \widetilde{S}_{m_{0}}, p \in \mathbb{R}^{N}$, $\|\varphi\|_{H^{1}} \leq \frac{1}{8} r_{*}$.

Then for $|q-p| \geq 2 R_{*}$ and $\tilde{U} \in \widetilde{S}_{m_{0}}$ we have

$$
\begin{aligned}
\|u-\tilde{U}(x-q)\|_{H^{1}\left(|x-q| \leq R_{*}\right)} \geq & \|\tilde{U}(x-q)\|_{H^{1}\left(|x-q| \leq R_{*}\right)} \\
& -\|U(x-p)\|_{H^{1}\left(|x-p| \geq R_{*}\right)}-\frac{1}{8} r_{*} \\
> & \frac{3}{4} r_{*}-\frac{1}{8} r_{*}-\frac{1}{8} r_{*}=\frac{1}{2} r_{*} .
\end{aligned}
$$

Thus $d(q, u)=0$ for $|q-p| \geq 2 R_{*}$. We can also see that, for small $r>0$

$$
d(q, u)=1 \text { for }|q-p|<r .
$$

Thus $B(p, r) \subset \operatorname{supp} d(\cdot, u) \subset B\left(p, 2 R_{*}\right)$. Therefore $\beta(u)$ is well-defined and we have

$$
\beta(u) \in B\left(p, 2 R_{*}\right) \quad \text { for } \quad u \in \widehat{S}\left(\frac{1}{8} r_{*}\right) .
$$


Shift equivariance and locally Lipschitz continuity of $\beta$ can be checked easily. Setting $\nu_{2}=\frac{1}{8} r_{*}$ and $R_{0}=2 R_{*}$, we have the desired result.

Using this lemma we have

Lemma 3.6 For $\delta_{0}>0$ given in Corollary 3.3, we have for $\varepsilon>0$ small

$$
J_{\varepsilon}\left(\omega\left(\frac{x-p / \varepsilon}{s}\right)\right) \geq E\left(m_{0}\right)+2 \delta_{0} .
$$

for all $\omega \in S_{m_{0}}, s \in\left[1-s_{0}, 1+s_{0}\right], p \in K_{\frac{3}{2} d_{0}} \backslash K_{\frac{1}{2} d_{0}}$.

Proof. For $\omega \in S_{m_{0}}, s \in\left[1-s_{0}, 1+s_{0}\right]$, we have

$$
\begin{aligned}
J_{\varepsilon}\left(\omega\left(\frac{x-p / \varepsilon}{s}\right)\right) & =L_{V(p)}(\omega(x / s))+\frac{1}{2} \int_{\mathbb{R}^{N}}(V(\varepsilon x+p)-V(p)) \omega(x / s)^{2} d x \\
& \geq L_{m_{0}+\rho_{0}}(\omega(x / s))+o(1),
\end{aligned}
$$

where $o(1)$ tends to zero as $\varepsilon \rightarrow 0$.

By (3.8), we have the desired result for $\varepsilon>0$ small.

It follows the corollary:

Lemma 3.7 There exists $\nu_{3}>0$ such that for $\varepsilon>0$ small

$$
J_{\varepsilon}(u) \geq E\left(m_{0}\right)+\delta_{0}
$$

for all $u \in \widehat{S}\left(\nu_{3}\right)$ with $\varepsilon \beta(u) \in K_{\frac{5}{4} d_{0}} \backslash K_{\frac{3}{4} d_{0}}$.

Proof. For $u(x)=\omega\left(\frac{x-p / \varepsilon}{s}\right)+\varphi(x)$ with $\|\varphi\|_{H^{1}}<\nu_{2}$, we have

$$
|\varepsilon \beta(u)-p| \leq \varepsilon\left|\beta(u)-\frac{p}{\varepsilon}\right| \leq \varepsilon R_{0} .
$$

Thus $\varepsilon \beta(u) \rightarrow p$ as $\varepsilon \rightarrow 0$ and for $\varepsilon>0$ small, $\varepsilon \beta(u) \in K_{\frac{5}{4} d_{0}} \backslash K_{\frac{3}{4} d_{0}}$ implies $p \in K_{\frac{3}{2} d_{0}} \backslash K_{\frac{1}{2} d_{0}}$. Since

$$
J_{\varepsilon}(u)-J_{\varepsilon}\left(\omega\left(\frac{x-p / \varepsilon}{s}\right)\right) \rightarrow 0
$$

uniformly as $\|\varphi\|_{H^{1}} \rightarrow 0$, Lemma 3.6 implies the conclusion of Lemma 3.7 for small $\nu_{3}>0$. 


\section{Gradient estimates}

For $\nu>0$ small, we set

$$
N_{\varepsilon}(\nu)=\left\{u \in \widehat{S}(\nu) ; \varepsilon \beta(u) \in K_{d_{0}}\right\} .
$$

We give the following $\varepsilon$-dependent concentration-compactness type result, which will give a useful gradient estimate later in Corollary 4.2.

Proposition 4.1 For sufficiently small $\nu_{4}>0, J_{\varepsilon}(u)$ has the following property: Let $\left(\varepsilon_{j}\right) \subset(0,1)$ be a sequence such that $\varepsilon_{j} \rightarrow 0$ as $j \rightarrow+\infty$ and let $\left(u_{j}\right) \subset H^{1}\left(\mathbb{R}^{N}\right)$ be such that $u_{j} \in N_{\varepsilon_{j}}\left(\nu_{4}\right)$ and

$$
\begin{aligned}
& J_{\varepsilon_{j}}\left(u_{j}\right) \rightarrow E\left(m_{0}\right), \\
& J_{\varepsilon_{j}}^{\prime}\left(u_{j}\right) \rightarrow 0 \text { as } j \rightarrow+\infty .
\end{aligned}
$$

Then there exist, up to a subsequence, $\left(p_{j}\right) \subset \mathbb{R}^{N}, p_{0} \in K$ and $\omega_{0} \in S_{m_{0}}$ such that $p_{j} \rightarrow p_{0}$ and

$$
\left\|u_{j}\left(\cdot+\frac{p_{j}}{\varepsilon_{j}}\right)-\omega_{0}\right\|_{H^{1}} \rightarrow 0 \quad \text { as } j \rightarrow+\infty
$$

Proof. Choose $\nu \in\left(0, \min \left\{\nu_{0}, \nu_{1}, \nu_{2}, \nu_{3}\right\}\right)$ such that

$$
\nu<\frac{1}{2} \min \left\{\|\omega(x / s)\|_{H^{1}\left(B\left(0, R_{0}\right)\right)} ; \omega \in S_{m_{0}}, s \in\left[1-s_{0}, 1+s_{0}\right]\right\} .
$$

Suppose that $u_{j} \in N_{\varepsilon_{j}}(\nu)$ satisfies (4.1)-(4.2). Setting $p_{j}=\varepsilon_{j} \beta\left(u_{j}\right) \in K_{d_{0}}$, we have for some $p_{0} \in \overline{K_{d_{0}}}$ and $u_{0} \in H^{1}\left(\mathbb{R}^{N}\right)$

$$
\begin{aligned}
& u_{j}\left(x+\frac{p_{j}}{\varepsilon_{j}}\right) \rightarrow u_{0}(x) \neq 0 \quad \text { weakly in } H^{1}\left(\mathbb{R}^{N}\right), \\
& p_{j} \rightarrow p_{0} \in \overline{K_{d_{0}}} .
\end{aligned}
$$

We will show that $u_{j}\left(x+\frac{p_{j}}{\varepsilon_{j}}\right) \rightarrow u_{0}(x)$ strongly in $H^{1}\left(\mathbb{R}^{N}\right)$ and $p_{0} \in K$.

Step 1: For large $n \in \mathbb{N}$ and $j \in \mathbb{N}$, there exists $k_{j}^{n} \in\{1,2, \cdots, n\}$ such that

$$
\left\|u_{j}\right\|_{H^{1}\left(A_{k_{j}^{n}}^{n}\right)}^{2} \leq \frac{4 \nu^{2}}{n},
$$


where

$$
A_{k}^{n}=\left\{x \in \mathbb{R}^{N} ; n k \leq\left|x-\frac{p_{j}}{\varepsilon_{j}}\right| \leq n(k+1)\right\} .
$$

In fact, for large $n$ we may assume $\left\|u_{j}\right\|_{H^{1}\left(B\left(\frac{p_{j}}{\varepsilon_{j}}, n\right)^{c}\right)} \leq 2 \nu$. Since

$$
\sum_{k=1}^{n}\left\|u_{j}\right\|_{H^{1}\left(A_{k}^{n}\right)}^{2} \leq\left\|u_{j}\right\|_{H^{1}\left(B\left(\frac{p_{j}}{\varepsilon_{j}}, n\right)^{c}\right)}^{2} \leq 4 \nu^{2},
$$

we can find a $k_{j}^{n} \in\{1,2, \cdots, n\}$ with the desired property.

For $k_{j}^{n}$ given in Step 1, we set

$$
v_{j}^{n}(x)=\widetilde{\zeta}_{n\left(k_{j}^{n}+1\right)}\left(\left|x-\frac{p_{j}}{\varepsilon_{j}}\right|\right) u_{j}(x),
$$

where $\widetilde{\zeta}_{R}(s)$ is given in (2.4).

Step 2: $\limsup _{j \rightarrow \infty}\left|J_{\varepsilon}^{\prime}\left(v_{j}^{n}\right) v_{j}^{n}\right| \leq C_{n}$, where $C_{n}>0$ satisfies $C_{n} \rightarrow 0$ as $n \rightarrow \infty$.

We compute

$$
\begin{aligned}
& \left(J_{\varepsilon_{j}}^{\prime}\left(u_{j}\right)-J_{\varepsilon_{j}}^{\prime}\left(v_{j}^{n}\right)\right) v_{j}^{n} \\
= & \int_{\mathbb{R}^{N}}\left(\nabla u_{j}-\nabla v_{j}^{n}\right) \nabla v_{j}^{n}+V\left(\varepsilon_{j} x\right)\left(u_{j}-v_{j}^{n}\right) v_{j}^{n} d x-\frac{1}{2}\left(\mathcal{D}^{\prime}\left(u_{j}\right)-\mathcal{D}^{\prime}\left(v_{j}^{n}\right)\right) v_{j}^{n} \\
= & (I)+(I I) .
\end{aligned}
$$

Clearly,

$$
|(I)| \leq C\left\|u_{j}\right\|_{H^{1}\left(A_{k_{j}^{n}}^{n}\right)}^{2} \leq C \frac{4 \nu^{2}}{n}
$$

By Corollary 2.6 (iii),

$$
|(I I)| \leq C_{1}\left\|u_{j}\right\|_{H^{1}\left(A_{k_{j}^{n}}^{n}\right)}^{\frac{N+\alpha}{N}}+C_{2} m_{n} \leq C_{1}\left(\frac{4 \nu^{2}}{n}\right)^{\frac{N+\alpha}{2 N}}+C_{2} m_{n} .
$$

Since $J_{\varepsilon_{j}}^{\prime}\left(u_{j}\right) \rightarrow 0$, we have Step 2 .

Step 3: $\limsup _{j \rightarrow \infty}\left\|u_{j}\left(x+\frac{p_{j}}{\varepsilon_{j}}\right)\right\|_{H^{1}\left(B(0, n(n+1))^{c}\right)} \leq C_{n}^{\prime}$, where $C_{n}^{\prime}>0$ satisfies $C_{n}^{\prime} \rightarrow 0$ as $n \rightarrow \infty$.

By Corollary 2.3, we have

$$
\limsup _{j \rightarrow \infty}\left\|v_{j}^{n}\right\|_{H^{1}} \leq \frac{1}{c_{0}} C_{n} .
$$


Since $\left\|u_{j}\right\|_{H^{1}\left(B\left(\frac{p_{j}}{\varepsilon_{j}}, n(n+1)\right)^{c}\right)} \leq\left\|v_{j}^{n}\right\|_{H^{1}}$, we have Step 3 .

Step 4: $u_{j}\left(x+\frac{p_{j}}{\varepsilon_{j}}\right) \rightarrow u_{0}$ strongly in $H^{1}\left(\mathbb{R}^{N}\right)$.

Set $w_{j}(x)=u_{j}\left(x+\frac{p_{j}}{\varepsilon_{j}}\right)$. Since $w_{j} \rightarrow u_{0}$ weakly in $H^{1}\left(\mathbb{R}^{N}\right)$, we have $w_{j} \rightarrow u_{0}$ strongly in $L_{l o c}^{q}\left(\mathbb{R}^{N}\right)$ for any $q \in\left[2, \frac{2 N}{N-2}\right)$. By Step 3 , we have

$$
\lim _{n \rightarrow \infty} \limsup _{j \rightarrow \infty}\left\|w_{j}\right\|_{L^{q}\left(B(0, n(n+1))^{c}\right)}=0
$$

for any $q \in\left[2, \frac{2 N}{N-2}\right]$. Thus we have $w_{j} \rightarrow u_{0}$ strongly in $L^{q}\left(\mathbb{R}^{N}\right)$ for any $q \in\left[2, \frac{2 N}{N-2}\right)$, which implies

$$
\mathcal{D}^{\prime}\left(w_{j}\right) \rightarrow \mathcal{D}^{\prime}\left(u_{0}\right) \text { strongly in } H^{-1}\left(\mathbb{R}^{N}\right) .
$$

We compute

$$
\begin{aligned}
& \left\|\nabla w_{j}\right\|_{2}^{2}+V\left(p_{0}\right)\left\|w_{j}\right\|_{2}^{2}=\left\|\nabla w_{j}\right\|_{2}^{2}+\int_{\mathbb{R}^{N}} V\left(\varepsilon_{j} x+p_{j}\right) w_{j}^{2} d x+o(1) \\
= & \left\|\nabla u_{j}\right\|_{2}^{2}+\int_{\mathbb{R}^{N}} V\left(\varepsilon_{j} x\right) u_{j}^{2} d x+o(1)=J_{\varepsilon_{j}}^{\prime}\left(u_{j}\right) u_{j}+\frac{1}{2} \mathcal{D}^{\prime}\left(u_{j}\right) u_{j}+o(1) \\
= & \frac{1}{2} \mathcal{D}^{\prime}\left(u_{j}\right) u_{j}+o(1)=\frac{1}{2} \mathcal{D}^{\prime}\left(w_{j}\right) w_{j}+o(1) \\
= & \frac{1}{2} \mathcal{D}^{\prime}\left(u_{0}\right) u_{0} .
\end{aligned}
$$

Similarly,

$$
\begin{aligned}
& \left\|\nabla u_{0}\right\|_{2}^{2}+V\left(p_{0}\right)\left\|u_{0}\right\|_{2}^{2} \\
= & \int_{\mathbb{R}^{N}} \nabla w_{j} \nabla u_{0}+V\left(p_{0}\right) w_{j} u_{0} d x+o(1) \\
= & \int_{\mathbb{R}^{N}} \nabla w_{j} \nabla u_{0}+V\left(\varepsilon_{j} x+p_{0}\right) w_{j} u_{0} d x+o(1) \\
= & \int_{\mathbb{R}^{N}} \nabla u_{j} \nabla u_{0}\left(x-\frac{p_{j}}{\varepsilon_{j}}\right)+V\left(\varepsilon_{j} x\right) u_{j} u_{0}\left(x-\frac{p_{j}}{\varepsilon_{j}}\right) d x+o(1) \\
= & J_{\varepsilon_{j}}^{\prime}\left(u_{j}\right) u_{0}\left(x-\frac{p_{j}}{\varepsilon_{j}}\right)+\frac{1}{2} \mathcal{D}^{\prime}\left(u_{j}\right) u_{0}\left(x-\frac{p_{j}}{\varepsilon_{j}}\right)+o(1) \\
= & \frac{1}{2} \mathcal{D}^{\prime}\left(u_{j}\right) u_{0}\left(x-\frac{p_{j}}{\varepsilon_{j}}\right)+o(1) \\
= & \frac{1}{2} \mathcal{D}^{\prime}\left(w_{j}\right) u_{0}+o(1) \\
= & \frac{1}{2} \mathcal{D}^{\prime}\left(u_{0}\right) u_{0} .
\end{aligned}
$$


Thus we have $\left\|\nabla w_{j}\right\|_{2}^{2}+V\left(p_{0}\right)\left\|w_{j}\right\|_{2}^{2} \rightarrow\left\|\nabla u_{0}\right\|_{2}^{2}+V\left(p_{0}\right)\left\|u_{0}\right\|_{2}^{2}$, which implies the conclusion of Step 4 .

Step 5: Conclusion.

We show that $p \in K$ and $u_{0}$ is a least energy solution of

$$
-\Delta u+m_{0} u=\left(I_{\alpha} * F(u)\right) f(u) \text { in } \mathbb{R}^{N} .
$$

By (4.2), we have for any $\varphi \in C_{0}^{\infty}\left(\mathbb{R}^{N}, \mathbb{R}\right)$

$$
J_{\varepsilon_{j}}^{\prime}\left(u_{j}\right) \varphi\left(x-\frac{p_{j}}{\varepsilon_{j}}\right) \rightarrow 0,
$$

from which we have

$$
\int_{\mathbb{R}^{N}} \nabla u_{0} \nabla \varphi+V\left(p_{0}\right) u_{0} \varphi d x-\frac{1}{2} \mathcal{D}^{\prime}\left(u_{0}\right) \varphi=0 .
$$

Thus $u_{0}$ is a solution of (4.3). We also have

$$
J_{\varepsilon_{j}}\left(u_{j}\right) \rightarrow \frac{1}{2}\left\|\nabla u_{0}\right\|_{2}^{2}+\frac{1}{2} V\left(p_{0}\right)\left\|u_{0}\right\|_{2}^{2}-\frac{1}{2} \mathcal{D}\left(u_{0}\right)
$$

and we have $E\left(m_{0}\right)=\frac{1}{2}\left\|\nabla u_{0}\right\|_{2}^{2}+\frac{1}{2} V\left(p_{0}\right)\left\|u_{0}\right\|_{2}^{2}-\frac{1}{2} \mathcal{D}\left(u_{0}\right)$. Recalling $p_{0} \in \overline{K_{d_{0}}}$ and $\inf _{x \in \overline{K_{d_{0}}}} V(x)=m_{0}$, we have $p_{0} \in K$ and $E\left(m_{0}\right)=L_{m_{0}}\left(u_{0}\right)$.

The following corollary gives an uniform estimate of $\left\|J_{\varepsilon}^{\prime}(u)\right\|_{H^{-1}}$ in an annular neighborhood of a set of expected solutions, which is one of the keys of our argument.

Corollary 4.2 Let $\nu_{4}>0$ be given in Proposition 4.1. Then for any $0<$ $\rho_{1}<\rho_{2}<\nu_{4}$ and for all $d \in\left(0, d_{0}\right)$ there exists $\delta_{1}>0$ such that for $\varepsilon>0$ small

$$
\left\|J_{\varepsilon}^{\prime}(u)\right\|_{H^{-1}} \geq \delta_{1}
$$

for all $u \in N_{\varepsilon}\left(\nu_{4}\right)$ with $J_{\varepsilon}(u) \in\left[E\left(m_{0}\right)-\delta_{1}, E\left(m_{0}\right)+\delta_{1}\right]$ and $(\widehat{\rho}(u), \varepsilon \beta(u)) \in$ $\left(\left[0, \rho_{2}\right] \times K_{d_{0}}\right) \backslash\left(\left[0, \rho_{1}\right] \times K_{d}\right)$.

Proof. By contradiction, we assume that there exist $\left(\varepsilon_{j}\right) \subset(0,1]$ such that $\varepsilon_{j} \rightarrow 0$, as $j \rightarrow+\infty$ and $\left(u_{j}\right) \subset H^{1}\left(\mathbb{R}^{N}\right)$ such that

$$
\begin{aligned}
& u_{j} \in N_{\varepsilon_{j}}\left(\nu_{4}\right), \\
& \left(\widehat{\rho}\left(u_{j}\right), \varepsilon \beta\left(u_{j}\right)\right) \in\left(\left[0, \rho_{2}\right] \times K_{d_{0}}\right) \backslash\left(\left[0, \rho_{1}\right] \times K_{d}\right) .
\end{aligned}
$$


and

$$
J_{\varepsilon_{j}}\left(u_{j}\right) \rightarrow E\left(m_{0}\right), \quad J_{\varepsilon_{j}}^{\prime}\left(u_{j}\right) \rightarrow 0 \quad \text { as } j \rightarrow+\infty .
$$

By Proposition 4.1, there exist, up to a subsequence, $\left(p_{j}\right) \subset \mathbb{R}^{N}, p \in K$ and $\omega_{0} \in S_{m_{0}}$ such that $p_{j} \rightarrow p$ and

$$
\left\|u_{j}\left(\cdot+\frac{p_{j}}{\varepsilon_{j}}\right)-\omega_{0}\right\|_{H^{1}} \rightarrow 0 \quad \text { as } j \rightarrow+\infty .
$$

Therefore we have

$$
\left\|u_{j}-\omega_{0}\left(\cdot-\frac{p_{j}}{\varepsilon_{j}}\right)\right\|_{H^{1}} \rightarrow 0 \quad \text { as } j \rightarrow+\infty
$$

and thus $\varepsilon_{j} \beta\left(u_{j}\right) \rightarrow p \in K$ and $\widehat{\rho}\left(u_{j}\right) \rightarrow 0$. A contradiction follows.

Finally we need to prove the following result.

Proposition 4.3 For any $\varepsilon \in(0,1]$ fixed, the Palais-Smale condition holds for $J_{\varepsilon}$ in the set $N_{\varepsilon}\left(\nu_{4}\right)$. That is, if a sequence $\left(u_{j}\right) \subset N_{\varepsilon}\left(\nu_{4}\right)$ satisfies

$$
\begin{aligned}
& J_{\varepsilon}\left(u_{j}\right) \rightarrow c, \\
& J_{\varepsilon}^{\prime}\left(u_{j}\right) \rightarrow 0 \text { as } j \rightarrow+\infty,
\end{aligned}
$$

for some constant $c \in \mathbb{R}$, then $\left(u_{j}\right)$ has a strong convergent subsequence in $H^{1}\left(\mathbb{R}^{N}\right)$.

Proof. Let $\varepsilon>0$ be fixed and a sequence $\left(u_{j}\right) \subset N_{\varepsilon}\left(\nu_{4}\right)$ satisfy (4.4)(4.5). Since $N_{\varepsilon}\left(\nu_{4}\right)$ is bounded in $H^{1}\left(\mathbb{R}^{N}\right)$, we can assume that $\left(u_{j}\right)$ weakly converges in $H^{1}\left(\mathbb{R}^{N}\right)$ to some $u_{0} \in H^{1}\left(\mathbb{R}^{N}\right)$, up to subsequences.

As in Steps 1-3 of the proof of Proposition 4.1, we can show

$$
\lim _{n \rightarrow \infty} \limsup _{j \rightarrow \infty}\left\|u_{j}\right\|_{H^{1}\left(B\left(\beta\left(u_{j}\right), n(n+1)\right)^{c}\right)}=0 .
$$

We note that $\beta\left(u_{j}\right)$ stays bounded as $j \rightarrow \infty$, since $\varepsilon \beta\left(u_{j}\right) \in K_{d_{0}}$ and $\varepsilon \in(0,1]$ is fixed. Thus we have

$$
\lim _{R \rightarrow \infty} \limsup _{j \rightarrow \infty}\left\|u_{j}\right\|_{H^{1}\left(B(0, R)^{c}\right)}=0
$$

which implies $u_{j} \rightarrow u_{0}$ strongly in $H^{1}\left(\mathbb{R}^{N}\right)$. 


\section{Deformation outside small balls}

We set

$$
\nu_{*}=\frac{1}{6} \min \left\{\nu_{0}, \nu_{1}, \cdots, \nu_{4}\right\}>0 .
$$

In this section, we construct a map $\tau_{\varepsilon}: N_{\varepsilon}\left(\nu_{*}\right) \rightarrow N_{\varepsilon}\left(5 \nu_{*}\right)$ whose properties are given in the following proposition.

Proposition 5.1 For sufficiently small $\varepsilon>0$, there exists a map $\tau_{\varepsilon}: N_{\varepsilon}\left(\nu_{*}\right) \rightarrow N_{\varepsilon}\left(5 \nu_{*}\right)$ such that

(i) $\tau_{\varepsilon}: N_{\varepsilon}\left(\nu_{*}\right) \rightarrow N_{\varepsilon}\left(5 \nu_{*}\right)$ is continuous.

(ii) $\tau_{\varepsilon}(u)=u$ if $u(x)=0$ for all $|x-\beta(u)| \geq \frac{1}{\sqrt{\varepsilon}}$.

(iii) For all $u \in N_{\varepsilon}\left(\nu_{*}\right)$,

$$
\begin{aligned}
& \tau_{\varepsilon}(u)(x)=u(x) \quad \text { for } x \in B\left(\beta(u), \frac{1}{\sqrt{\varepsilon}}\right), \\
& J_{\varepsilon}\left(\tau_{\varepsilon}(u)\right) \leq J_{\varepsilon}(u) \\
& \left\|\tau_{\varepsilon}(u)\right\|_{H^{1}\left(B\left(\beta(u), \frac{2}{\sqrt{\varepsilon}}\right)^{c}\right)} \leq \widetilde{\rho}_{\varepsilon} \\
& \left|\beta\left(\tau_{\varepsilon}(u)\right)-\beta(u)\right| \leq 2 R_{0}, \\
& \widehat{\rho}\left(\tau_{\varepsilon}(u)\right) \leq 5 \nu_{*} .
\end{aligned}
$$

Here $\widetilde{\rho}_{\varepsilon}>0$ is independent of $u$ and satisfies $\widetilde{\rho}_{\varepsilon} \rightarrow 0$ as $\varepsilon \rightarrow 0$.

We note that this type of operators were introduced in Byeon and Tanaka [10, 11, 12] for nonlinear Schrödinger equations and a related problem through

a minimizing problem outside small balls, which is related to the following boundary value problem:

$$
\begin{aligned}
& -\Delta v+V(\varepsilon x) v=f(v) \quad \text { in } B\left(\beta(u), \frac{1}{\sqrt{\varepsilon}}\right)^{c}, \\
& v=u \quad \text { on } \partial B\left(\beta(u), \frac{1}{\sqrt{\varepsilon}}\right), \\
& \|v\|_{H^{1}\left(B\left(\beta(u), \frac{1}{\sqrt{\varepsilon}}\right)^{c}\right)} \leq 5 \nu_{*} .
\end{aligned}
$$

That is, $\tau_{\varepsilon}(u)$ is given as a solution of the above boundary value problem. In this argument, to obtain unique solvability and continuity of the operator, 
strict convexity of the corresponding functional is important. Because of nonlocal nonlinearity, it can be verified for our problem just under the condition $\lim \sup _{t \rightarrow 0}\left|\frac{f(t)}{t}\right|<\infty$, that is, $p \geq 2$ for $f(s)=|s|^{p-2} s$.

Here we take another approach to construct $\tau_{\varepsilon}$ and it will be constructed using a special deformation flow outside small balls.

To make our construction of $\tau_{\varepsilon}$ clearly, we fix the center of balls and work in a fixed space $H^{1}\left(B\left(0, \frac{1}{\sqrt{\varepsilon}}\right)^{c}\right)$. First we note

$$
J_{\varepsilon}(u)=\widetilde{J}_{\varepsilon}(\varepsilon \beta(u), u(x+\beta(u))),
$$

where

$$
\widetilde{J}_{\varepsilon}(p, v)=\frac{1}{2}\|\nabla v\|_{2}^{2}+\frac{1}{2} \int_{\mathbb{R}^{N}} V(\varepsilon x+p) v^{2} d x-\frac{1}{2} \mathcal{D}(v) .
$$

We denote restriction of $u(x+\beta(u))$ on the set $B\left(0, \frac{1}{\sqrt{\varepsilon}}\right)^{c}$ by $\Theta_{\varepsilon}(u)$. We note that for all $u \in N_{\varepsilon}\left(\nu_{*}\right)$

$\Theta_{\varepsilon}(u) \in Y_{\varepsilon} \equiv\left\{v \in H^{1}\left(B\left(0, \frac{1}{\sqrt{\varepsilon}}\right)^{c}\right) ;\|v\|_{H^{1}\left(B\left(0, \frac{1}{\sqrt{\varepsilon}}\right)^{c}\right)}<\frac{3}{2} \nu_{*}\right\} \quad$ for $\varepsilon>0$ small.

In what follows, we construct a vector field $V(v): Y_{\varepsilon} \rightarrow H_{0}^{1}\left(B\left(0, \frac{1}{\sqrt{\varepsilon}}\right)^{c}\right)$ with special properties. We define $K_{1 \varepsilon}(v), K_{2 \varepsilon}(v): H^{1}\left(B\left(0, \frac{1}{\sqrt{\varepsilon}}\right)^{c}\right) \rightarrow \mathbb{R}$ by

$$
K_{1 \varepsilon}(v)=\|v\|_{H^{1}\left(B\left(0, \frac{1}{\sqrt{\varepsilon}}\right)^{c}\right)}^{2}, \quad K_{2 \varepsilon}(v)=\|v\|_{H^{1}\left(B\left(0, \frac{2}{\sqrt{\varepsilon}}\right)^{c}\right)}^{2},
$$

which will play important roles to construct $\tau_{\varepsilon}$. We also set $M=\sup _{\omega \in S_{m_{0}}, s \in\left[1-s_{0}, 1+s_{0}\right]}\|\omega(x / s)\|_{H^{1}}+1$.

Lemma 5.2 For any $v \in Y_{\varepsilon}$, there exists a $\mathcal{V}_{v} \in H_{0}^{1}\left(B\left(0, \frac{1}{\sqrt{\varepsilon}}\right)^{c}\right)$ such that

$$
\left\|\mathcal{V}_{v}\right\|_{H^{1}} \leq 2 \nu_{*}
$$

(ii) If $u \in H^{1}\left(\mathbb{R}^{N}\right)$ satisfies

$$
\|u\|_{H^{1}} \leq M \quad \text { and } \quad u=v \text { in } B\left(0, \frac{1}{\sqrt{\varepsilon}}\right)^{c},
$$

then for any $p \in K_{2 d_{0}}$

$$
\widetilde{J}_{\varepsilon}^{\prime}(p, u) \mathcal{V}_{v} \geq a\left(\|v\|_{H^{1}\left(B\left(0, \frac{2}{\sqrt{\varepsilon}}\right)^{c}\right)}^{2}-\rho_{\varepsilon}\right) .
$$


Here $a>0$ is independent of $\varepsilon, p, u, v$ and $\rho_{\varepsilon}$ is independent of $p, u$, $v$ and satisfies

$$
\rho_{\varepsilon} \rightarrow 0 \quad \text { as } \varepsilon \rightarrow 0
$$

(iii) For any $v \in Y_{\varepsilon}$,

$$
K_{1 \varepsilon}^{\prime}(v) \mathcal{V}_{v}, K_{2 \varepsilon}^{\prime}(v) \mathcal{V}_{v} \geq a\left(\|v\|_{H^{1}\left(B\left(0, \frac{2}{\sqrt{\varepsilon}}\right)^{c}\right)}^{2}-\rho_{\varepsilon}\right)
$$

Here $a>0$ and $\rho_{\varepsilon}>0$ are as in (ii).

Proof. Proof is divided into 3 steps.

We denote by $n_{\varepsilon}$ the largest integer less than $1 / \varepsilon^{1 / 4}$.

Step 1: For any $v \in Y_{\varepsilon}$, there exists $k \in\left\{1,2, \cdots, n_{\varepsilon}\right\}$ such that

$$
\|v\|_{H^{1}\left(|x| \in\left[\frac{1}{\sqrt{\varepsilon}}+\frac{k-1}{\varepsilon^{1 / 4}}, \frac{1}{\sqrt{\varepsilon}}+\frac{k}{\varepsilon^{1 / 4}}\right]\right)}^{2}<\left(\frac{3}{2}\right)^{2} \frac{\nu_{*}^{2}}{n_{\varepsilon}} .
$$

In fact, we have

$$
\sum_{k=1}^{n_{\varepsilon}}\|v\|_{H^{1}\left(|x| \in\left[\frac{1}{\sqrt{\varepsilon}}+\frac{k-1}{\varepsilon^{1 / 4}}, \frac{1}{\sqrt{\varepsilon}}+\frac{k}{\varepsilon^{1 / 4}}\right]\right)}^{2} \leq\|v\|_{H^{1}\left(B\left(0, \frac{1}{\sqrt{\varepsilon}}\right)^{c}\right)}^{2} \leq\left(\frac{3}{2}\right)^{2} \nu_{*}^{2} .
$$

Thus Step 1 holds.

For a $k$ given in Step 1, we set

$$
\mathcal{V}_{v}(x)=\widetilde{\zeta}_{\frac{1}{\sqrt{\varepsilon}}+\frac{k}{\varepsilon^{1 / 4}}}(|x|) v(x) \in H_{0}^{1}\left(B\left(0, \frac{1}{\sqrt{\varepsilon}}\right)^{c}\right) .
$$

Here $\widetilde{\zeta}_{R}(s)$ is defined in (2.4) . For $\varepsilon>0$ small, clearly we have the property (5.7).

Step 2: $\mathcal{V}_{v}$ satisfies (5.9). 
We compute

$$
\begin{aligned}
& \widetilde{J}_{\varepsilon}^{\prime}(p, u) \mathcal{V}_{v} \\
= & \int_{\mathbb{R}^{N}} \nabla v \nabla\left(\widetilde{\zeta}_{\frac{1}{\sqrt{\varepsilon}}+\frac{k}{\varepsilon^{1 / 4}}} v\right) d x+\int_{\mathbb{R}^{N}} V(\varepsilon x+p) \widetilde{\zeta}_{\frac{1}{\sqrt{\varepsilon}}+\frac{k}{\varepsilon^{1 / 4}}} v^{2} d x \\
& -\frac{1}{2} \mathcal{D}^{\prime}(u)\left(\widetilde{\zeta}_{\frac{1}{\sqrt{\varepsilon}}+\frac{k}{\varepsilon^{1 / 4}}} v\right) \\
= & \int_{|x|>\frac{1}{\sqrt{\varepsilon}}+\frac{k}{\varepsilon^{1 / 4}}}|\nabla v|^{2}+V(\varepsilon x+p) v^{2} d x \\
& +\int_{|x| \in\left[\frac{1}{\sqrt{\varepsilon}}+\frac{k-1}{\varepsilon^{1 / 4}}, \frac{1}{\sqrt{\varepsilon}}+\frac{k}{\varepsilon^{1 / 4}}\right]} \nabla v \nabla\left(\widetilde{\zeta}_{\frac{1}{\sqrt{\varepsilon}}+\frac{k}{\varepsilon^{1 / 4}}} v\right)+V(\varepsilon x+p) \widetilde{\zeta}_{\frac{1}{\sqrt{\varepsilon}}+\frac{k}{\varepsilon^{1 / 4}}} v^{2} d x \\
& -\frac{1}{2} \mathcal{D}^{\prime}(u)\left(\widetilde{\zeta}_{\frac{1}{\sqrt{\varepsilon}}+\frac{k}{\varepsilon^{1 / 4}}} v\right) \\
\geq & c\|v\|_{H^{1}\left(|x|>\frac{1}{\sqrt{\varepsilon}}+\frac{k}{\varepsilon^{1 / 4}}\right)}^{2}+(I)-(I I),
\end{aligned}
$$

where $c=\min \{1, \underline{V}\}$.

We can easily see that

$$
|(I)| \leq c^{\prime}\|v\|_{H^{1}\left(|x| \in\left[\frac{1}{\sqrt{\varepsilon}}+\frac{k-1}{\varepsilon^{1 / 4}}, \frac{1}{\sqrt{\varepsilon}}+\frac{k}{\varepsilon^{1 / 4}}\right]\right)}^{2} \leq C^{\prime} \frac{\nu_{*}^{2}}{n_{\varepsilon}} .
$$

By Corollary 2.6 (iii),

$$
\begin{array}{ll} 
& \mid(I I)-\mathcal{D}^{\prime}\left(\widetilde{\zeta}_{\frac{1}{\sqrt{\varepsilon}}+\frac{k}{\varepsilon^{1 / 4}}} v\right)\left(\widetilde{\zeta}_{\left.\frac{1}{\sqrt{\varepsilon}}+\frac{k}{\varepsilon^{1 / 4}} v\right) \mid} \leq \quad C_{1}\|v\|_{H^{1}\left(|x| \in\left[\frac{1}{\sqrt{\varepsilon}}+\frac{k-1}{\varepsilon^{1 / 4}}, \frac{1}{\sqrt{\varepsilon}}+\frac{k}{\varepsilon^{1 / 4}}\right]\right)}^{\frac{N+\alpha}{2}}+C_{2} m_{1 / \varepsilon^{1 / 4}}\right. \\
\leq \quad & C_{1}\left(\left(\frac{3}{2}\right)^{2} \frac{\nu_{*}^{2}}{n_{\varepsilon}}\right)^{\frac{N+\alpha}{2 N}}+C_{2} m_{1 / \varepsilon^{1 / 4}} .
\end{array}
$$


By Proposition 2.1, using $\sigma(t)$ defined in (2.3), we have

$$
\begin{aligned}
& \left|\mathcal{D}^{\prime}\left(\widetilde{\zeta}_{\frac{1}{\sqrt{\varepsilon}}+\frac{k}{\varepsilon^{1 / 4}}} v\right)\left(\widetilde{\zeta}_{\frac{1}{\sqrt{\varepsilon}}+\frac{k}{\varepsilon^{1 / 4}}} v\right)\right| \\
\leq & C\left\|F\left(\widetilde{\zeta}_{\frac{1}{\sqrt{\varepsilon}}+\frac{k}{\varepsilon^{1 / 4}}} v\right)\right\|_{\frac{2 N}{N+\alpha}}\left\|f\left(\widetilde{\zeta}_{\frac{1}{\sqrt{\varepsilon}}+\frac{k}{\varepsilon^{1 / 4}}} v\right)\left(\widetilde{\zeta}_{\frac{1}{\sqrt{\varepsilon}}+\frac{k}{\varepsilon^{1 / 4}}} v\right)\right\|_{\frac{2 N}{N+\alpha}} \\
\leq & C^{\prime} \sigma\left(\left\|\widetilde{\zeta}_{\frac{1}{\sqrt{\varepsilon}}+\frac{k}{\varepsilon^{1 / 4}}} v\right\|_{H^{1}}\right)^{\frac{N+\alpha}{N}} \\
\leq & C^{\prime \prime} \sigma\left(\|v\|_{H^{1}\left(|x|>\frac{1}{\sqrt{\varepsilon}}+\frac{k}{\varepsilon^{1 / 4}}\right)}\right)^{\frac{N+\alpha}{N}}+C^{\prime \prime \prime} \sigma\left(\|v\|_{H^{1}\left(|x| \in\left[\frac{1}{\sqrt{\varepsilon}}+\frac{k-1}{\varepsilon^{1 / 4}}, \frac{1}{\sqrt{\varepsilon}}+\frac{k}{\varepsilon^{1 / 4}}\right]\right)}\right)^{\frac{N+\alpha}{N}} \\
\leq & C^{\prime \prime} \sigma\left(\|v\|_{H^{1}\left(|x|>\frac{1}{\sqrt{\varepsilon}}+\frac{k}{\varepsilon^{1 / 4}}\right)}\right)^{\frac{N+\alpha}{N}}+C^{\prime \prime \prime} \sigma\left(\sqrt{\frac{\nu_{*}^{2}}{n_{\varepsilon}}}\right)^{\frac{N+\alpha}{N}} .
\end{aligned}
$$

Choosing $\nu_{4}>0$ smaller if necessary, we may assume that for some $a>0$

$$
c t^{2}-C^{\prime \prime} \sigma(t)^{\frac{N+\alpha}{N}} \geq a t^{2} \quad \text { for } t \in\left[0, \frac{3}{2} \nu_{*}\right]
$$

Thus, we have

$$
\begin{aligned}
\widetilde{J}_{\varepsilon}^{\prime}(p, u) \mathcal{V}_{v} & \geq a\|v\|_{H^{1}\left(|x|>\frac{1}{\sqrt{\varepsilon}}+\frac{k}{\varepsilon^{1 / 4}}\right)}^{2}-c_{\varepsilon} \\
& \geq a\|v\|_{H^{1}\left(|x|>\frac{2}{\sqrt{\varepsilon}}\right)}^{2}-c_{\varepsilon},
\end{aligned}
$$

where $c_{\varepsilon}=C^{\prime} \frac{\nu_{*}^{2}}{n_{\varepsilon}}+C_{1}\left(\left(\frac{3}{2}\right)^{2} \frac{\nu_{*}^{2}}{n_{\varepsilon}}\right)^{\frac{N+\alpha}{N}}+C^{\prime \prime \prime} \sigma\left(\sqrt{\frac{\nu_{*}^{2}}{n_{\varepsilon}}}\right)^{\frac{N+\alpha}{N}}+C_{2} m_{1 / \varepsilon^{1 / 4}}$. Setting $\rho_{\varepsilon}=\frac{1}{a} c_{\varepsilon}$, we have (5.9).

Step 3: $\mathcal{V}_{v}$ satisfies (5.10).

Since

$$
\begin{aligned}
& K_{1 \varepsilon}^{\prime}(v) \mathcal{V}_{v}=2 \int_{B\left(0, \frac{1}{\sqrt{\varepsilon}}\right)^{c}} \nabla v \nabla\left(\widetilde{\zeta}_{\frac{1}{\sqrt{\varepsilon}}+\frac{k}{\varepsilon^{1 / 4}}} v\right)+\widetilde{\zeta}_{\frac{1}{\sqrt{\varepsilon}}+\frac{k}{\varepsilon^{1 / 4}}} v^{2} d x \\
& K_{2 \varepsilon}^{\prime}(v) \mathcal{V}_{v}=2 \int_{B\left(0, \frac{2}{\sqrt{\varepsilon}}\right)^{c}}|\nabla v|^{2}+v^{2} d x,
\end{aligned}
$$

(5.10) can be shown as in Step 2.

We define

$$
\begin{aligned}
\widehat{Y}_{\varepsilon} & =\left\{v \in Y_{\varepsilon} ; K_{2 \varepsilon}(v)>3 \rho_{\varepsilon}\right\} \\
& =\left\{v \in H^{1}\left(B\left(0, \frac{1}{\sqrt{\varepsilon}}\right)^{c}\right) ;\|v\|_{H^{1}\left(B\left(0, \frac{1}{\sqrt{\varepsilon}}\right)^{c}\right)}<\frac{3}{2} \nu_{*},\|v\|_{H^{1}\left(B\left(0, \frac{2}{\sqrt{\varepsilon}}\right)^{c}\right)}^{2}>3 \rho_{\varepsilon}\right\} .
\end{aligned}
$$

Setting $\widetilde{\mathcal{V}}_{v}=\mathcal{V}_{v} /\left\|\mathcal{V}_{v}\right\|_{H^{1}}$, we have 
Lemma 5.3 For any $v \in \widehat{Y}_{\varepsilon}$, there exists $\widetilde{\mathcal{V}}_{v} \in H_{0}^{1}\left(B\left(0, \frac{1}{\sqrt{\varepsilon}}\right)^{c}\right)$ such that

(i) $\left\|\widetilde{\mathcal{V}}_{v}\right\|_{H^{1}} \leq 1$

(ii) $\widetilde{J}_{\varepsilon}^{\prime}(p, u) \widetilde{\mathcal{V}}_{v}, K_{1 \varepsilon}^{\prime}(v) \widetilde{\mathcal{V}}_{v}, K_{2 \varepsilon}^{\prime}(v) \widetilde{\mathcal{V}}_{v}>\frac{a \rho_{\varepsilon}}{\nu_{*}}$ for $u \in H^{1}\left(\mathbb{R}^{N}\right)$ with (5.8) and $p \in K_{2 d_{0}}$.

Clearly for any $v \in \widehat{Y}_{\varepsilon}$, there exists an open neighborhood $U_{v}$ in $\widehat{Y}_{\varepsilon}$ such that

$$
\widetilde{J}_{\varepsilon}^{\prime}(p, \widehat{u}) \widetilde{\mathcal{V}}_{v}, K_{1 \varepsilon}^{\prime}(\widehat{v}) \widetilde{\mathcal{V}}_{v}, K_{2 \varepsilon}^{\prime}(\widehat{v}) \widetilde{\mathcal{V}}_{v}>\frac{a \rho_{\varepsilon}}{\nu_{*}}
$$

hold for $\widehat{v} \in U_{v}$, where $\widehat{u}$ and $\widehat{v}$ satisfies (5.8) and $p \in K_{2 d_{0}}$.

Using partition of unity, we have

Proposition 5.4 There exists a locally Lipschitz vector field $V(v): \widehat{Y}_{\varepsilon} \rightarrow$ $H_{0}^{1}\left(B\left(0, \frac{1}{\sqrt{\varepsilon}}\right)^{c}\right)$ such that for all $v \in \widehat{Y}_{\varepsilon}$

(i) $\|V(v)\|_{H^{1}} \leq 1$.

(ii) $\widetilde{J}_{\varepsilon}^{\prime}(p, u) V(v), K_{1 \varepsilon}^{\prime}(v) V(v), K_{2 \varepsilon}^{\prime}(v) V(v)>\frac{a \rho_{\varepsilon}}{\nu_{*}}$ for $u \in H^{1}\left(\mathbb{R}^{N}\right)$ with (5.8) and $p \in K_{2 d_{0}}$.

We choose a function $\varphi(r) \in C^{\infty}(\mathbb{R}, \mathbb{R})$ such that

$$
\begin{gathered}
\varphi(r)= \begin{cases}1 & \text { for } r \geq 4 \rho_{\varepsilon}, \\
0 & \text { for } r \leq 3 \rho_{\varepsilon},\end{cases} \\
\varphi(r) \in[0,1] \text { for all } r \in \mathbb{R} .
\end{gathered}
$$

We consider the following ODE in $Y_{\varepsilon}$ : for $v \in Y_{\varepsilon}$

$$
\left\{\begin{array}{l}
\frac{d w}{d \tau}=-\varphi\left(K_{2 \varepsilon}(w)\right) V(w), \\
w(0, v)=v
\end{array}\right.
$$

Lemma 5.5 Let $w(\tau, v)$ be the unique solution of (5.11). Then

(i) $w(\tau, v) \in Y_{\varepsilon}$ for all $\tau \geq 0$ and $v \in Y_{\varepsilon}$. 
(ii) Let $v \in Y_{\varepsilon}$ and suppose that $u \in H^{1}\left(\mathbb{R}^{N}\right)$ satisfies (5.8) and $p \in K_{2 d_{0}}$. Then

$$
\frac{d}{d \tau} \widetilde{J}_{\varepsilon}\left(p, \widetilde{w}_{u}(\tau, v)\right) \leq 0,
$$

where

$$
\widetilde{w}_{u}(\tau, v)(x)= \begin{cases}u(x) & \text { for } x \in B\left(0, \frac{1}{\sqrt{\varepsilon}}\right), \\ w(\tau, v) & \text { for } x \in B\left(0, \frac{1}{\sqrt{\varepsilon}}\right)^{c} .\end{cases}
$$

(iii) For $i=1,2$,

$$
\begin{aligned}
& \frac{d}{d \tau} K_{i \varepsilon}(w(\tau, v)) \leq 0 \quad \text { for all } v \in Y_{\varepsilon}, \\
& \frac{d}{d \tau} K_{i \varepsilon}(w(\tau, v)) \leq-\frac{a \rho_{\varepsilon}}{\nu_{*}} \quad \text { if } K_{2 \varepsilon}(w(\tau, v)) \geq 4 \rho_{\varepsilon} .
\end{aligned}
$$

(iv) There exists $T_{\varepsilon}>0$ such that for all $v \in Y_{\varepsilon}$

$$
K_{2 \varepsilon}\left(w\left(T_{\varepsilon}, v\right)\right) \leq 4 \rho_{\varepsilon} .
$$

Proof. First we show (iii). Noting that $\varphi\left(K_{2 \varepsilon}(w)\right)>0$ implies $\|w\|_{H^{1}\left(B\left(0, \frac{1}{\sqrt{\varepsilon}}\right)^{c}\right)}>3 \rho_{\varepsilon}$, that is, $w \in \widehat{Y}_{\varepsilon}$, we have from Proposition 5.4

$$
\begin{aligned}
\frac{d}{d \tau} K_{i \varepsilon}(w(\tau, v)) & =-\varphi\left(K_{2 \varepsilon}(w)\right) K_{i \varepsilon}^{\prime}(w) V(w) \\
& \leq-\frac{a \rho_{\varepsilon}}{\nu_{*}} \varphi\left(K_{2 \varepsilon}(w)\right) .
\end{aligned}
$$

Thus, (iii) holds. By (iii), $K_{1 \varepsilon}(w(\tau, v))=\|w(\tau, u)\|_{H^{1}\left(B\left(0, \frac{1}{\sqrt{\varepsilon}}\right)^{c}\right)}^{2}$ is nonincreasing. Thus $w(\tau, v)$ exists and satisfies $w(\tau, v) \in Y_{\varepsilon}$ for all $\tau \in[0, \infty)$. That is, $w(\tau, v):[0, \infty) \times Y_{\varepsilon} \rightarrow Y_{\varepsilon}$ is well-defined. Thus (i) follows.

(iv) follows from (iii) easily. Noting $\left.w(\tau, v)\right|_{|x|=\frac{1}{\sqrt{\varepsilon}}}$ is independent of $\tau$, we have $\widetilde{w}_{u}(\tau, v) \in H^{1}\left(\mathbb{R}^{N}\right)$. Therefore (ii) follows from Proposition 5.4 (ii).

Now we define for $u \in N_{\varepsilon}\left(\nu_{*}\right)$

$$
W(\tau, u)(x)= \begin{cases}u(x) & \text { for } x \in B\left(\beta(u), \frac{1}{\sqrt{\varepsilon}}\right) . \\ w\left(\tau, \Theta_{\varepsilon}(u)\right)(x-\beta(u)) & \text { for } x \in B\left(\beta(u), \frac{1}{\sqrt{\varepsilon}}\right)^{c}\end{cases}
$$


and

$$
\begin{aligned}
\widehat{W}(\tau, u)(x) & =W(\tau, u)(x+\beta(u)) \\
& = \begin{cases}u(x+\beta(u)) & \text { for } x \in B\left(0, \frac{1}{\sqrt{\varepsilon}}\right), \\
w\left(\tau, \Theta_{\varepsilon}(u)\right)(x) & \text { for } x \in B\left(0, \frac{1}{\sqrt{\varepsilon}}\right)^{c} .\end{cases}
\end{aligned}
$$

We have

Lemma 5.6 For $\varepsilon>0$ small, we have

(i) For any $\tau \in[0, \infty)$,

$$
\begin{aligned}
& W(\tau, u)(x)=u(x) \quad \text { for } x \in B\left(\beta(u), \frac{1}{\sqrt{\varepsilon}}\right), \\
& J_{\varepsilon}(W(\tau, u)) \leq J_{\varepsilon}(u), \\
& \|W(\tau, u)\|_{H^{1}\left(B\left(\beta(u), \frac{1}{\sqrt{\varepsilon}}\right)^{c}\right)} \leq 2 \nu_{*}, \\
& |\beta(W(\tau, u))-\beta(u)| \leq 2 R_{0}, \\
& \widehat{\rho}(W(\tau, u)) \leq 5 \nu_{*} .
\end{aligned}
$$

(ii) Moreover we have

$$
\left\|W\left(T_{\varepsilon}, u\right)\right\|_{H^{1}\left(B\left(\beta(u), \frac{2}{\sqrt{\varepsilon}}\right)^{c}\right)} \leq 2 \sqrt{\rho_{\varepsilon}} .
$$

Proof. (i) Obviously we have (5.13).

We have for $u \in N_{\varepsilon}\left(\nu_{*}\right)$

$$
\begin{aligned}
J_{\varepsilon}(W(\tau, u)) & =\widetilde{J}_{\varepsilon}(\varepsilon \beta(u), W(\tau, u)(x+\beta(u))) \\
& =\widetilde{J}_{\varepsilon}(\varepsilon \beta(u), \widehat{W}(\tau, u)(x))
\end{aligned}
$$

Thus we deduce (5.14) from (ii) of Lemma 5.5.

For (5.15), we note that

$$
\|u\|_{H^{1}\left(B\left(\beta(u), \frac{1}{\sqrt{\varepsilon}}\right)^{c}\right)}<2 \nu_{*} \quad \text { for } \varepsilon>0 \text { small. }
$$

Since

$$
\|W(\tau, u)\|_{H^{1}\left(B\left(\beta(u), \frac{1}{\sqrt{\varepsilon}}\right)^{c}\right)}^{2}=\|\widehat{W}(\tau, u)\|_{H^{1}\left(B\left(0, \frac{1}{\sqrt{\varepsilon}}\right)^{c}\right)}^{2}=K_{1 \varepsilon}\left(w\left(\tau, \Theta_{\varepsilon}(u)\right)\right)
$$


is non-increasing by (iii) of Lemma 5.5, we have (5.15).

Next we show (5.16) and (5.17). We write $u \in N_{\varepsilon}\left(\nu_{*}\right)$ as $u(x)=\omega\left(\frac{x-p}{s}\right)+$ $\varphi(x)$, where $\omega \in S_{m_{0}}, p \in \mathbb{R}^{N}, s \in\left[1-s_{0}, 1+s_{0}\right]$ and $\|\varphi\|_{H^{1}}<\nu_{*}$. By Lemma 3.5, we have

$$
|\beta(u)-p| \leq R_{0}
$$

Since

$$
\begin{gathered}
\|W(\tau, u)-u\|_{H^{1}}=\|W(\tau, u)-u\|_{H^{1}\left(B\left(\beta(u), \frac{1}{\sqrt{\varepsilon}}\right)^{c}\right)} \\
\leq\left\|w\left(\tau, \Theta_{\varepsilon}(u)\right)\right\|_{H^{1}\left(B\left(0, \frac{1}{\sqrt{\varepsilon}}\right)^{c}\right)}+\|u\|_{H^{1}\left(B\left(\beta(u), \frac{1}{\sqrt{\varepsilon}}\right)^{c}\right)} \leq 4 \nu_{*},
\end{gathered}
$$

we have

$$
\left\|W(\tau, u)-\omega\left(\frac{x-p}{s}\right)\right\|_{H^{1}} \leq\|W(\tau, u)-u\|_{H^{1}}+\left\|u-\omega\left(\frac{x-p}{s}\right)\right\|_{H^{1}} \leq 5 \nu_{*} .
$$

Thus by our choice of $\nu_{*}$, we have

$$
|\beta(W(\tau, u))-p| \leq R_{0}
$$

from which and (5.18) we have (5.16). Clearly (5.17) follows from (5.19).

(ii) Since

$$
\|W(\tau, u)\|_{H^{1}\left(B\left(\beta(u), \frac{2}{\sqrt{\varepsilon}}\right)^{c}\right)}^{2}=\left\|w\left(\tau, \Theta_{\varepsilon}(u)\right)\right\|_{H^{1}\left(B\left(0, \frac{2}{\sqrt{\varepsilon}}\right)^{c}\right)}^{2}=K_{2 \varepsilon}\left(w\left(\tau, \Theta_{\varepsilon}(u)\right)\right),
$$

(ii) follows from (iv) of Lemma 5.5,

Now we can give a proof to Proposition 5.1.

Proof of Proposition 5.1. We set for $u \in N_{\varepsilon}\left(\nu_{*}\right)$

$$
\tau_{\varepsilon}(u)=W\left(T_{\varepsilon}, u\right) .
$$

The desired properties (5.2) $-(\underline{5.6})$ with $\widetilde{\rho}_{\varepsilon}=2 \sqrt{\rho_{\varepsilon}}$ follow from Lemma 5.6 .

\section{An invariant set and a deformation flow}

This section is devoted to develop a deformation argument. To this aim we introduce a neighborhood $\mathcal{X}_{\varepsilon}$ of the set of expected solutions, which is positively invariant under a pseudo-gradient flow. 


\subsection{A pseudo-gradient flow}

For $\nu_{*}>0$ defined in (5.1), we fix $\rho_{1}, \rho_{2}>0$ such that $0<\rho_{1}<\rho_{2}<\nu_{*}$ and we choose $\delta_{0}, \delta_{1}>0$ by Lemma 3.7 and Corollary 4.2 with $d=\frac{1}{2} d_{0}$. We also set

$$
\delta_{*}=\min \left\{\frac{\delta_{1}}{4}\left(\rho_{2}-\rho_{1}\right), \delta_{0}\right\}>0 .
$$

With these choices of $\nu_{*}, \delta_{*}$ we have for $\varepsilon>0$ small

(i) $\Lambda(u)$ and $\beta(u)$ are well-defined on $N_{\varepsilon}\left(6 \nu_{*}\right)$ and satisfy

$$
\begin{aligned}
& \Lambda(u) \in\left[1-2 s_{0}, 1+2 s_{0}\right], \\
& \varepsilon \beta(u) \in K_{2 d_{0}}
\end{aligned}
$$

for all $u \in N_{\varepsilon}\left(6 \nu_{*}\right)$.

(ii) By Lemma 2.2, for all $u(x)=\omega\left(\frac{x-p}{s}\right)+\varphi(x)$ with $\omega \in S_{m_{0}}, s \in$ $\left[1-s_{0}, 1+s_{0}\right],\|\varphi\|_{H^{1}}<6 \nu_{*}$

$$
|\beta(u)-p|<R_{0}
$$

(iii) By Lemma 3.7

$$
J_{\varepsilon}(u) \geq E\left(m_{0}\right)+\delta_{0}
$$

if $u \in N_{\varepsilon}\left(6 \nu_{*}\right)$ satisfies $\varepsilon \beta(u) \in K_{\frac{5}{4} d_{0} \backslash K_{\frac{3}{4}} d_{0}}$.

(iv) By Corollary 4.2,

$$
\left\|J_{\varepsilon}^{\prime}(u)\right\|_{H^{-1}} \geq \delta_{1},
$$

if $u \in N_{\varepsilon}\left(6 \nu_{*}\right)$ satisfies $J_{\varepsilon}(u) \in\left[E\left(m_{0}\right)-\delta_{1}, E\left(m_{0}\right)+\delta_{1}\right]$ and $(\widehat{\rho}(u), \varepsilon \beta(u)) \in\left(\left[0, \rho_{2}\right] \times K_{d_{0}}\right) \backslash\left(\left[0, \rho_{1}\right] \times K_{\frac{1}{2} d_{0}}\right)$.

We define

$$
\mathcal{X}_{\varepsilon}=\left\{u \in N_{\varepsilon}\left(\nu_{*}\right) \mid J_{\varepsilon}(u) \leq E\left(m_{0}\right)+\delta_{*}-\frac{\delta_{1}}{2}\left(\widehat{\rho}(u)-\rho_{1}\right)_{+}\right\} .
$$

We shall try to find critical points of $J_{\varepsilon}$ in $\mathcal{X}_{\varepsilon}$. We note that

(a) By (6.1), for $u \in N_{\varepsilon}\left(6 \nu_{*}\right), \varepsilon \beta(u) \notin K_{\frac{3}{4} d_{0}}$ implies

$$
J_{\varepsilon}(u) \geq E\left(m_{0}\right)+\delta_{0}>E\left(m_{0}\right)+\delta_{*},
$$

which implies $u \notin \mathcal{X}_{\varepsilon}$. Thus we have

$$
\varepsilon \beta(u) \in K_{\frac{3}{4} d_{0}} \quad \text { for all } u \in \mathcal{X}_{\varepsilon} .
$$


(b) For $u \in \mathcal{X}_{\varepsilon}, \widehat{\rho}(u) \geq \rho_{2}$ implies

$$
J_{\varepsilon}(u) \leq E\left(m_{0}\right)+\delta_{*}-\frac{\delta_{1}}{2}\left(\rho_{2}-\rho_{1}\right)<E\left(m_{0}\right)-\delta_{*} .
$$

The following deformation result plays an essential role to show the existence of critical points.

Proposition 6.1 For any $c \in\left(E\left(m_{0}\right)-\delta_{*}, E\left(m_{0}\right)+\delta_{*}\right)$ and for any neighborhood $U$ of $\mathcal{K}_{c} \equiv\left\{u \in \mathcal{X}_{\varepsilon} ; J_{\varepsilon}^{\prime}(u)=0, J_{\varepsilon}(u)=c\right\}\left(U=\emptyset\right.$ if $\left.\mathcal{K}_{c}=\emptyset\right)$, there exist $r>0$ with $(c-r, c+r) \subset\left(E\left(m_{0}\right)-\delta_{*}, E\left(m_{0}\right)+\delta_{*}\right)$ and a deformation $\eta(\tau, u):[0,1] \times\left(\mathcal{X}_{\varepsilon} \backslash U\right) \rightarrow \mathcal{X}_{\varepsilon}$ such that

(i) $\eta(0, u)=u$ for all $u$.

(ii) $\eta(\tau, u)=u$ for all $\tau \in[0,1]$ if $J_{\varepsilon}(u) \notin\left[E\left(m_{0}\right)-\delta_{*}, E\left(m_{0}\right)+\delta_{*}\right]$.

(iii) $J_{\varepsilon}(\eta(\tau, u))$ is a non-increasing function of $\tau$ for all $u$.

(iv) $J_{\varepsilon}(\eta(1, u)) \leq c-r$ for all $u \in \mathcal{X}_{\varepsilon} \backslash U$ satisfying $J_{\varepsilon}(u) \leq c+r$.

Proof. We consider a deformation flow defined by

$$
\left\{\begin{array}{l}
\frac{d \eta}{d \tau}=-\phi(\eta) \frac{\mathcal{V}(\eta)}{\|\mathcal{V}(\eta)\|_{H^{1}}} \\
\eta(0, u)=u
\end{array}\right.
$$

where $\mathcal{V}(u):\left\{u \in H^{1}\left(\mathbb{R}^{N}\right) ; J_{\varepsilon}^{\prime}(u) \neq 0\right\} \rightarrow H^{1}\left(\mathbb{R}^{N}\right)$ is a locally Lipschitz continuous pseudo-gradient vector field satisfying

$$
\|\mathcal{V}(u)\|_{H^{1}} \leq\left\|J_{\varepsilon}^{\prime}(u)\right\|_{H^{-1}}, \quad J_{\varepsilon}^{\prime}(u) \mathcal{V}(u) \geq \frac{1}{2}\left\|J_{\varepsilon}^{\prime}(u)\right\|_{H^{-1}}^{2}
$$

and $\phi(u): H^{1}\left(\mathbb{R}^{N}\right) \rightarrow[0,1]$ is a locally Lipschitz continuous function, which is defined in the standard way. We require that $\phi(u)$ satisfies

$$
\phi(u)= \begin{cases}0 & \text { if } J_{\varepsilon}(u) \notin\left[E\left(m_{0}\right)-\delta_{*}, E\left(m_{0}\right)+\delta_{*}\right] . \\ 1 & \text { if } J_{\varepsilon}(u) \in[c-\kappa, c+\kappa] \backslash \mathcal{N},\end{cases}
$$

where $[c-\kappa, c+\kappa] \subset\left(E\left(m_{0}\right)-\delta_{*}, E\left(m_{0}\right)+\delta_{*}\right)$ and $\mathcal{N}$ is an open set such that $\mathcal{K}_{c} \subset \mathcal{N} \subset U$. 
We consider the flow defined by (6.6). The properties (i)-(iii) follows by standard arguments from the definition (6.6) and since $\phi(u)=0$ if $J_{\varepsilon}(u) \notin$ $\left[E\left(m_{0}\right)-\delta_{*}, E\left(m_{0}\right)+\delta_{*}\right]$. Clearly also since, by Proposition 4.3, $J_{\varepsilon}$ satisfies the Palais-Smale condition for fixed $\varepsilon>0$, property (iv) is standard. Thus to end the proof we just need to show that

$$
\eta\left(\tau, \mathcal{X}_{\varepsilon}\right) \subset \mathcal{X}_{\varepsilon} \quad \text { for all } \tau \geq 0
$$

namely that $\mathcal{X}_{\varepsilon}$ is positively invariant under our flow. First note that because of property (iii), 6.3 implies that for $u \in \mathcal{X}_{\varepsilon}, \eta(t)=\eta(t, u)$ remains in the set $\left\{u ; \varepsilon \beta(u) \in K_{\frac{3}{4} d_{0}}\right\}$. Also, because of property (ii) and (6.5), for $u \in \mathcal{X}_{\varepsilon}$, $\eta(t)$ remains in $N_{\varepsilon}\left(\nu_{*}\right)$. Thus to show (6.7), we just need to prove that the property

$$
J_{\varepsilon}(u) \leq E\left(m_{0}\right)+\delta_{*}-\frac{\delta_{1}}{2}\left(\widehat{\rho}(u)-\rho_{1}\right)_{+}
$$

is stable under the deformation. For this it suffices to show that for a solution $\eta(\tau)$ of (6.6) , if $0<s<t<1$ satisfies

$$
\begin{gathered}
\widehat{\rho}(\eta(\tau)) \in\left[\rho_{1}, \rho_{2}\right] \quad \text { for all } \tau \in[s, t], \\
J_{\varepsilon}(\eta(s)) \leq E\left(m_{0}\right)+\delta_{*}-\frac{\delta_{1}}{2}\left(\widehat{\rho}(\eta(s))-\rho_{1}\right),
\end{gathered}
$$

then

$$
J_{\varepsilon}(\eta(t)) \leq E\left(m_{0}\right)+\delta_{*}-\frac{\delta_{1}}{2}\left(\widehat{\rho}(\eta(t))-\rho_{1}\right) .
$$

We note that $(\widehat{\rho}(\eta(\tau)), \varepsilon \beta(\eta(\tau))) \in\left[\rho_{1}, \rho_{2}\right] \times K_{d_{0}} \subset\left(\left[0, \rho_{2}\right] \times K_{d_{0}}\right) \backslash\left(\left[0, \rho_{1}\right] \times\right.$ $\left.K_{\frac{1}{2} d_{0}}\right)$ for all $\tau \in[s, t]$. Thus by Corollary 4.2 , we have for $\tau \in[s, t]$

$$
\begin{aligned}
\frac{d}{d \tau} J_{\varepsilon}(\eta(\tau)) & =J_{\varepsilon}^{\prime}(\eta) \frac{d \eta}{d \tau}=-\phi(\eta) J_{\varepsilon}^{\prime}(\eta) \frac{\mathcal{V}(\eta)}{\|\mathcal{V}(\eta)\|_{H^{1}}} \\
& \leq-\frac{1}{2}\left\|J_{\varepsilon}^{\prime}(\eta)\right\|_{H^{-1}} \leq-\phi(\eta) \frac{\delta_{1}}{2}
\end{aligned}
$$

and

$$
J_{\varepsilon}(\eta(t)) \leq J_{\varepsilon}(\eta(s))-\frac{\delta_{1}}{2} \int_{s}^{t} \phi(\eta(\tau)) d \tau .
$$

On the other hand,

$$
\|\eta(t)-\eta(s)\|_{H^{1}} \leq \int_{s}^{t}\left\|\frac{d \eta}{d \tau}\right\|_{H^{1}} d \tau \leq \int_{s}^{t} \phi(\eta(\tau)) d \tau .
$$


By (6.8)-(6.9), and using the fact that $|\widehat{\rho}(\eta(t))-\widehat{\rho}(\eta(s))| \leq\|\eta(t)-\eta(s)\|_{H^{1}}$, we have

$$
\begin{aligned}
J_{\varepsilon}(\eta(t)) & \leq J_{\varepsilon}(\eta(s))-\frac{\delta_{1}}{2}\|\eta(t)-\eta(s)\|_{H^{1}} \\
& \leq J_{\varepsilon}(\eta(s))-\frac{\delta_{1}}{2}|\widehat{\rho}(\eta(t))-\widehat{\rho}(\eta(s))| \\
& \leq E\left(m_{0}\right)+\delta_{*}-\frac{\delta_{1}}{2}\left(\widehat{\rho}(\eta(s))-\rho_{1}\right)-\frac{\delta_{1}}{2}|\widehat{\rho}(\eta(t))-\widehat{\rho}(\eta(s))| \\
& \leq E\left(m_{0}\right)+\delta_{*}-\frac{\delta_{1}}{2}\left(\widehat{\rho}(\eta(t))-\rho_{1}\right) .
\end{aligned}
$$

Thus (6.7) holds and the proof of the proposition is completed.

Proposition 6.1 enables us to estimate the multiplicity of critical points using the relative category.

\subsection{Two maps $\Phi_{\varepsilon}$ and $\Psi_{\varepsilon}$}

For $a \in \mathbb{R}$, we set

$$
\mathcal{X}_{\varepsilon}^{a}=\left\{u \in \mathcal{X}_{\varepsilon} \mid J_{\varepsilon}(u) \leq a\right\} .
$$

For $\hat{\delta} \in\left(0, \delta_{*}\right)$ small, using relative category, we shall estimate the change of topology between $\mathcal{X}_{\varepsilon}^{E\left(m_{0}\right)+\hat{\delta}}$ and $\mathcal{X}_{\varepsilon}^{E\left(m_{0}\right)-\hat{\delta}}$. We introduce two maps:

$$
\begin{aligned}
\Phi_{\varepsilon}: & \left(\left[1-s_{0}, 1+s_{0}\right] \times K,\left\{1 \pm s_{0}\right\} \times K\right) \rightarrow\left(\mathcal{X}_{\varepsilon}^{E\left(m_{0}\right)+\hat{\delta}}, \mathcal{X}_{\varepsilon}^{E\left(m_{0}\right)-\hat{\delta}}\right), \\
\Psi_{\varepsilon}: & \left(\mathcal{X}_{\varepsilon}^{E\left(m_{0}\right)+\hat{\delta}}, \mathcal{X}_{\varepsilon}^{E\left(m_{0}\right)-\hat{\delta}}\right) \rightarrow \\
& \left(\left[1-s_{0}, 1+s_{0}\right] \times K_{d_{0}},\left(\left[1-s_{0}, 1+s_{0}\right] \backslash\{1\}\right) \times K_{d_{0}}\right) .
\end{aligned}
$$

Here we use notation from algebraic topology: $f:(A, B) \rightarrow\left(A^{\prime}, B^{\prime}\right)$ means $B \subset A, B^{\prime} \subset A^{\prime}, f: A \rightarrow A^{\prime}$ is continuous and $f(B) \subset B^{\prime}$.

Definition of $\Phi_{\varepsilon}$ :

We fix a positive least energy solution $\omega_{0}(x) \in S_{m_{0}}$ and set for $(s, p) \in$ $\left[1-s_{0}, 1+s_{0}\right] \times K$

$$
\Phi_{\varepsilon}(s, p)=\zeta_{\frac{1}{2 \sqrt{\varepsilon}}}\left(\left|x-\frac{p}{\varepsilon}\right|\right) \omega_{0}\left(\frac{x-\frac{p}{\varepsilon}}{s}\right) .
$$

Definition of $\Psi_{\varepsilon}$ :

We define $\Psi_{\varepsilon}$ as a composition of the following maps. 
(1) $N_{\varepsilon}\left(\nu_{*}\right) \rightarrow N_{\varepsilon}\left(6 \nu_{*}\right) ; u \mapsto \zeta_{\frac{2}{\sqrt{\varepsilon}}}(|x-\beta(u)|) \tau_{\varepsilon}(u)(x)$

(2) $N_{\varepsilon}\left(6 \nu_{*}\right) \rightarrow\left[1-s_{0}, 1+s_{0}\right] \times K_{d_{0}} ; v \mapsto(\psi(\Lambda(v)), \varepsilon \beta(v))$,

where $\tau_{\varepsilon}(u): N_{\varepsilon}\left(\nu_{*}\right) \rightarrow N_{\varepsilon}\left(5 \nu_{*}\right)$ is given in Proposition 5.1 and $\psi(s) \in$ $C(\mathbb{R}, \mathbb{R})$ is defined by

$$
\psi(s)= \begin{cases}1-s_{0} & \text { for } s<1-s_{0} \\ s & \text { for } 1-s_{0} \leq s \leq 1+s_{0} \\ 1+s_{0} & \text { for } s>1+s_{0}\end{cases}
$$

That is,

$$
\Psi_{\varepsilon}(u)=\left(\psi\left(\Lambda\left(\zeta_{\frac{2}{\sqrt{\varepsilon}}}(|x-\beta(u)|) \tau_{\varepsilon}(u)(x)\right)\right), \varepsilon \beta\left(\zeta_{\frac{2}{\sqrt{\varepsilon}}}(|x-\beta(u)|) \tau_{\varepsilon}(u)(x)\right)\right) .
$$

In what follows, we observe that $\Phi_{\varepsilon}$ and $\Psi_{\varepsilon}$ are well-defined for small $\hat{\delta}>0$.

For $(s, p) \in\left[1-s_{0}, 1+s_{0}\right] \times K$, we have as $\varepsilon \sim 0$

$$
\begin{aligned}
& \varepsilon \beta\left(\zeta_{\frac{1}{2 \sqrt{\varepsilon}}}\left(\left|x-\frac{p}{\varepsilon}\right|\right) \omega_{0}\left(\frac{x-\frac{p}{\varepsilon}}{s}\right)\right)=p+o(1) \\
& J_{\varepsilon}\left(\zeta_{\frac{1}{2 \sqrt{\varepsilon}}}\left(\left|x-\frac{p}{\varepsilon}\right|\right) \omega_{0}\left(\frac{x-\frac{p}{\varepsilon}}{s}\right)\right)=L_{m_{0}}\left(\omega_{0}\left(\frac{x-\frac{p}{\varepsilon}}{s}\right)\right)+o(1) \\
& \quad=\frac{1}{2} g(s)\left\|\nabla \omega_{0}\right\|_{2}^{2}+\frac{1}{2} h(s) m_{0}\left\|\omega_{0}\right\|_{2}^{2}+o(1) \\
& \quad=E\left(m_{0}\right)-\frac{1}{2}(g(1)-g(s))\left\|\nabla \omega_{0}\right\|_{2}^{2}-\frac{1}{2}(h(1)-h(s)) m_{0}\left\|\omega_{0}\right\|_{2}^{2}+o(1) .
\end{aligned}
$$

Here $g(s), h(s)$ are given in the proof of Lemma 3.1. Choosing $\hat{\delta}>0$ small so that

$$
2 \hat{\delta}<\frac{1}{2}\left(g(1)-g\left(1 \pm s_{0}\right)\right)\left\|\nabla \omega_{0}\right\|_{2}^{2}+\frac{1}{2}\left(h(1)-h\left(1 \pm s_{0}\right)\right) m_{0}\left\|\omega_{0}\right\|_{2}^{2},
$$

we find that $\Phi_{\varepsilon}$ is well-defined as a map $\left(\left[1-s_{0}, 1+s_{0}\right] \times K,\left\{1 \pm s_{0}\right\} \times K\right) \rightarrow$ $\left(\mathcal{X}_{\varepsilon}^{E\left(m_{0}\right)+\hat{\delta}}, \mathcal{X}_{\varepsilon}^{E\left(m_{0}\right)-\hat{\delta}}\right)$ for small $\varepsilon>0$.

Next we deal with $\Psi_{\varepsilon}$. For $u \in \mathcal{X}_{\varepsilon}^{E\left(m_{0}\right)+\hat{\delta}}$, we set $v_{\varepsilon}(x)=\zeta_{\frac{2}{\sqrt{\varepsilon}}}(\mid x-$ $\beta(u) \mid) \tau_{\varepsilon}(u)(x)$. By (iii) of Proposition 5.1,

$$
\begin{aligned}
J_{\varepsilon}(u) & \geq J_{\varepsilon}\left(\tau_{\varepsilon}(u)\right) \\
& =J_{\varepsilon}\left(\tau_{\frac{2}{\sqrt{\varepsilon}}}(|x-\beta(u)|) \tau_{\varepsilon}(u)\right)+o(1) \\
& =J_{\varepsilon}\left(v_{\varepsilon}\right)+o(1) .
\end{aligned}
$$


Here we use the fact that $\left\|\tau_{\varepsilon}(u)\right\|_{H^{1}\left(B\left(\beta(u), \frac{2}{\sqrt{\varepsilon}}\right)^{c}\right)} \leq \widetilde{\rho}_{\varepsilon} \rightarrow 0$ as $\varepsilon \rightarrow 0$ uniformly in $u$. Since $\operatorname{supp} v_{\varepsilon} \subset B\left(\beta(u), \frac{2}{\sqrt{\varepsilon}}+2\right)$ and $\lim _{\varepsilon \rightarrow 0} \max _{x \in B\left(\beta(u), \frac{2}{\sqrt{\varepsilon}}+2\right)} \mid V(\varepsilon x)-$ $V(\varepsilon \beta(u)) \mid=0$, we have

$$
\begin{aligned}
J_{\varepsilon}\left(v_{\varepsilon}\right) & =L_{V(\beta(u))}\left(v_{\varepsilon}\right)+o(1) \\
& \geq L_{m_{0}}\left(v_{\varepsilon}\right)+o(1) \\
& \geq E\left(m_{0}\right)\left(1-C_{0}\left(\Lambda\left(v_{\varepsilon}\right)-1\right)^{2}\right)+o(1) .
\end{aligned}
$$

Here we use Corollary 3.2. Thus, for $u \in \mathcal{X}_{\varepsilon}^{E\left(m_{0}\right)-\hat{\delta}}$,

$$
E\left(m_{0}\right)\left(1-C_{0}\left(\Lambda\left(v_{\varepsilon}\right)-1\right)^{2}\right) \leq E\left(m_{0}\right)-\hat{\delta}+o(1),
$$

which implies

$$
\Lambda\left(v_{\varepsilon}\right) \neq 1 \quad \text { for } \varepsilon>0 \text { small }
$$

and $\Psi_{\varepsilon}$ is well-defined as a map $\left(\mathcal{X}_{\varepsilon}^{E\left(m_{0}\right)+\hat{\delta}}, \mathcal{X}_{\varepsilon}^{E\left(m_{0}\right)-\hat{\delta}}\right) \rightarrow\left(\left[1-s_{0}, 1+s_{0}\right] \times\right.$ $\left.K_{2 d_{0}},\left(\left[1-s_{0}, 1+s_{0}\right] \backslash\{1\}\right) \times K_{2 d_{0}}\right)$. We also note that by (6.4),$\varepsilon \beta\left(v_{\varepsilon}\right) \in K_{\frac{3}{4} d_{0}}$. Thus $\Psi_{\varepsilon}$ is well-defined as a map

$$
\begin{aligned}
& \left(\mathcal{X}_{\varepsilon}^{E\left(m_{0}\right)+\hat{\delta}}, \mathcal{X}_{\varepsilon}^{E\left(m_{0}\right)-\hat{\delta}}\right) \\
& \rightarrow\left(\left[1-s_{0}, 1+s_{0}\right] \times K_{\frac{3}{4} d_{0}},\left(\left[1-s_{0}, 1+s_{0}\right] \backslash\{1\}\right) \times K_{\frac{3}{4} d_{0}}\right) \\
& \subset\left(\left[1-s_{0}, 1+s_{0}\right] \times K_{d_{0}},\left(\left[1-s_{0}, 1+s_{0}\right] \backslash\{1\}\right) \times K_{d_{0}}\right) .
\end{aligned}
$$

The next proposition will be important to estimate the relative category $\operatorname{cat}\left(\mathcal{X}_{\varepsilon}^{E\left(m_{0}\right)+\hat{\delta}}, \mathcal{X}_{\varepsilon}^{E\left(m_{0}\right)-\hat{\delta}}\right)$.

Proposition 6.2 For $\varepsilon>0$ small,

$$
\begin{aligned}
& \Psi_{\varepsilon} \circ \Phi_{\varepsilon}:\left(\left[1-s_{0}, 1+s_{0}\right] \times K,\left\{1 \pm s_{0}\right\} \times K\right) \\
& \quad \rightarrow\left(\left[1-s_{0}, 1+s_{0}\right] \times K_{d_{0}},\left(\left[1-s_{0}, 1+s_{0}\right] \backslash\{1\}\right) \times K_{d_{0}}\right)
\end{aligned}
$$

is homotopic to the embedding $j(s, p)=(s, p)$. That is, there exists a continuous map

$$
\eta:[0,1] \times\left[1-s_{0}, 1+s_{0}\right] \times K \rightarrow\left[1-s_{0}, 1+s_{0}\right] \times K_{d_{0}}
$$


such that

$$
\begin{aligned}
& \eta(0, s, p)=\left(\Psi_{\varepsilon} \circ \Phi_{\varepsilon}\right)(s, p), \\
& \eta(1, s, p)=(s, p) \quad \text { for all }(s, p) \in\left[1-s_{0}, 1+s_{0}\right] \times K, \\
& \eta(t, s, p) \in\left(\left[1-s_{0}, 1+s_{0}\right] \backslash\{1\}\right) \times K_{d} \\
& \quad \text { for all } t \in[0,1] \text { and }(s, p) \in\left\{1 \pm s_{0}\right\} \times K .
\end{aligned}
$$

Proof. For $(s, p) \in\left[1-s_{0}, 1+s_{0}\right] \times K$ and $\varepsilon>0$ small, we set

$$
w_{\varepsilon}(x)=\Phi_{\varepsilon}(s, p)(x)=\zeta_{\frac{1}{2 \sqrt{\varepsilon}}}\left(\left|x-\frac{p}{\varepsilon}\right|\right) \omega\left(\frac{x-\frac{p}{\varepsilon}}{s}\right) .
$$

We observe that $\left|\beta\left(w_{\varepsilon}\right)-\frac{p}{\varepsilon}\right| \leq R_{0}$. Thus $w_{\varepsilon}(x)=0$ in $B\left(\beta\left(w_{\varepsilon}\right), \frac{1}{\sqrt{\varepsilon}}\right)^{c}$ for $\varepsilon>0$ small, which implies $\tau_{\varepsilon}\left(w_{\varepsilon}\right)=w_{\varepsilon}$. Thus

$$
\begin{aligned}
\left(\Psi_{\varepsilon} \circ \Phi_{\varepsilon}\right)(s, p) & =\Psi_{\varepsilon}\left(w_{\varepsilon}\right)=\left(\psi\left(\Lambda\left(w_{\varepsilon}\right)\right), \varepsilon \beta\left(w_{\varepsilon}\right)\right) \\
& =(s, p)+o(1),
\end{aligned}
$$

which implies $\Psi_{\varepsilon} \circ \Phi_{\varepsilon}$ is homotopic to the embedding $j(s, p)=(s, p)$.

Differently from [17, 18], (see [15, Remark 4.3]) we can not infer in general that

$$
\operatorname{cat}\left(\mathcal{X}_{\varepsilon}^{E\left(m_{0}\right)+\hat{\delta}}, \mathcal{X}_{\varepsilon}^{E\left(m_{0}\right)-\hat{\delta}}\right) \geq \operatorname{cat}(K, \partial K) .
$$

Therefore in the work it will be necessary to use the notions of category and cup-length for maps.

\section{$7 \quad$ Proof of Theorem 1.4}

In order to prove our theorem, we shall need some topological tools that we now present for the reader convenience. Following [5], see also [23, 24], we define

Definition 7.1 Let $B \subset A$ and $B^{\prime} \subset A^{\prime}$ be topological spaces and $f$ : $(A, B) \rightarrow\left(A^{\prime}, B^{\prime}\right)$ be a continuous map, that is $f: A \rightarrow A^{\prime}$ is continuous and $f(B) \subset B^{\prime}$. The category $\operatorname{cat}(f)$ of $f$ is the least integer $k \geq 0$ such that there exist open sets $A_{0}, A_{1}, \cdots, A_{k}$ with the following properties:

(a) $A=A_{0} \cup A_{1} \cup \cdots \cup A_{k}$. 
(b) $B \subset A_{0}$ and there exists a map $h_{0}:[0,1] \times A_{0} \rightarrow A^{\prime}$ such that

$$
\begin{array}{ll}
h_{0}(0, x)=f(x) & \text { for all } x \in A_{0}, \\
h_{0}(1, x) \in B^{\prime} & \text { for all } x \in A_{0}, \\
h_{0}(t, x)=f(x) & \text { for all } x \in B \text { and } t \in[0,1] .
\end{array}
$$

(c) For $i=1,2, \cdots, k,\left.f\right|_{A_{i}}: A_{i} \rightarrow A^{\prime}$ is homotopic to a constant map.

We also introduce the cup-length of $f:(A, B) \rightarrow\left(A^{\prime}, B^{\prime}\right)$. Let $H^{*}$ denote Alexander-Spanier cohomology with coefficients in the field $\mathbb{F}$. We recall that the cup product $\smile$ turns $H^{*}(A)$ into a ring with unit $1_{A}$, and it turns $H^{*}(A, B)$ into a module over $H^{*}(A)$. A continuous map $f:(A, B) \rightarrow$ $\left(A^{\prime}, B^{\prime}\right)$ induces a homomorphism $f^{*}: H^{*}\left(A^{\prime}\right) \rightarrow H^{*}(A)$ of rings as well as a homomorphism $f^{*}: H^{*}\left(A^{\prime}, B^{\prime}\right) \rightarrow H^{*}(A, B)$ of abelian groups. We also use notation:

$$
\tilde{H}^{n}\left(A^{\prime}\right)= \begin{cases}0 & \text { for } n=0, \\ H^{n}\left(A^{\prime}\right) & \text { for } n>0 .\end{cases}
$$

For more details on algebraic topology we refer to [46].

Definition 7.2 For $f:(A, B) \rightarrow\left(A^{\prime}, B^{\prime}\right)$ the cup-length, $\operatorname{cupl}(f)$ is defined as follows; when $f^{*}: H^{*}\left(A^{\prime}, B^{\prime}\right) \rightarrow H^{*}(A, B)$ is not a trivial map, $\operatorname{cupl}(f)$ is defined as the maximal integer $k \geq 0$ such that there exist elements $\alpha_{1}, \cdots$, $\alpha_{k} \in \tilde{H}^{*}\left(A^{\prime}\right)$ and $\beta \in H^{*}\left(A^{\prime}, B^{\prime}\right)$ with

$$
\begin{aligned}
f^{*}\left(\alpha_{1} \smile \cdots \smile \alpha_{k} \smile \beta\right) & =f^{*}\left(\alpha_{1}\right) \smile \cdots \smile f^{*}\left(\alpha_{k}\right) \smile f^{*}(\beta) \\
& \neq 0 \text { in } H^{*}(A, B) .
\end{aligned}
$$

When $f^{*}=0: H^{*}\left(A^{\prime}, B^{\prime}\right) \rightarrow H^{*}(A, B)$, we define $\operatorname{cupl}(f)=-1$.

We note that $\operatorname{cupl}(f)=0$ if $f^{*} \neq 0: H^{*}\left(A^{\prime}, B^{\prime}\right) \rightarrow H^{*}(A, B)$ and $\tilde{H}^{*}\left(A^{\prime}\right)=0$.

As fundamental properties of $\operatorname{cat}(f)$ and $\operatorname{cupl}(f)$, we have

Proposition $7.3 \quad$ (i) For $f:(A, B) \rightarrow\left(A^{\prime}, B^{\prime}\right)$, $\operatorname{cat}(f) \geq \operatorname{cupl}(f)+1$.

(ii) For $f:(A, B) \rightarrow\left(A^{\prime}, B^{\prime}\right), f^{\prime}:\left(A^{\prime}, B^{\prime}\right) \rightarrow\left(A^{\prime \prime}, B^{\prime \prime}\right)$,

$$
\operatorname{cupl}\left(f^{\prime} \circ f\right) \leq \min \left\{\operatorname{cupl}\left(f^{\prime}\right), \operatorname{cupl}(f)\right\} .
$$


(iii) If $f, g:(A, B) \rightarrow\left(A^{\prime}, B^{\prime}\right)$ are homotopic, then $\operatorname{cupl}(f)=\operatorname{cupl}(g)$.

The proof of these statements can be found in [5, Lemmas 2.6, 2.7]. Finally we recall

Definition 7.4 For a set $(A, B)$, we define the relative category cat $(A, B)$ and the relative cup-length $\operatorname{cupl}(A, B)$ by

$$
\begin{aligned}
& \operatorname{cat}(A, B)=\operatorname{cat}\left(i d_{(A, B)}:(A, B) \rightarrow(A, B)\right), \\
& \operatorname{cupl}(A, B)=\operatorname{cupl}\left(i d_{(A, B)}:(A, B) \rightarrow(A, B)\right) .
\end{aligned}
$$

We also set

$$
\operatorname{cat}(A)=\operatorname{cat}(A, \emptyset), \quad \operatorname{cupl}(A)=\operatorname{cupl}(A, \emptyset)
$$

We also recall the following topological lemma due to T. Bartsch (cf. [15, Lemma 5.5]) where we make use of the continuity property of AlexanderSpanier cohomology.

Lemma 7.5 Let $K \subset \mathbb{R}^{N}$ be a compact set. For a d-neighborhood $K_{d}=$ $\left\{x \in \mathbb{R}^{N} ; \operatorname{dist}(x, K) \leq d\right\}$ and $I=[0,1], \partial I=\{0,1\}$, we consider the inclusion

$$
j:(I \times K, \partial I \times K) \rightarrow\left(I \times K_{d}, \partial I \times K_{d}\right)
$$

defined by $j(s, x)=(s, x)$. Then for $d>0$ small,

$$
\operatorname{cupl}(j) \geq \operatorname{cupl}(K)
$$

Now we have all the ingredients to give the

Proof of Theorem 1.4. We observe that for $\varepsilon>0$ small

$$
\#\left\{u \in \mathcal{X}_{\varepsilon}^{E\left(m_{0}\right)+\hat{\delta}} \backslash \mathcal{X}_{\varepsilon}^{E\left(m_{0}\right)-\hat{\delta}} ; J_{\varepsilon}^{\prime}(u)=0\right\} \geq \operatorname{cat}\left(\mathcal{X}_{\varepsilon}^{E\left(m_{0}\right)+\hat{\delta}}, \mathcal{X}_{\varepsilon}^{E\left(m_{0}\right)-\hat{\delta}}\right) .
$$

Using Proposition 6.1, (17.1) can be proved in a standard way (c.f. [23, Theorem 4.2]).

By (i) of Proposition 7.3, we have

$$
\operatorname{cat}\left(\mathcal{X}_{\varepsilon}^{E\left(m_{0}\right)+\hat{\delta}}, \mathcal{X}_{\varepsilon}^{E\left(m_{0}\right)-\hat{\delta}}\right) \geq \operatorname{cupl}\left(\mathcal{X}_{\varepsilon}^{E\left(m_{0}\right)+\hat{\delta}}, \mathcal{X}_{\varepsilon}^{E\left(m_{0}\right)-\hat{\delta}}\right)+1
$$


Since $\Psi_{\varepsilon} \circ \Phi_{\varepsilon}=\Psi_{\varepsilon} \circ i d_{\left(\mathcal{X}_{\varepsilon}^{E\left(m_{0}\right)+\hat{\delta}}, \mathcal{X}_{\varepsilon}^{\left.E\left(m_{0}\right)-\hat{\delta}\right)}\right.} \circ \Phi_{\varepsilon}$, it follows from (ii) of Proposition 7.3 that

$$
\begin{aligned}
\operatorname{cupl}\left(\Psi_{\varepsilon} \circ \Phi_{\varepsilon}\right) & \leq \operatorname{cupl}\left(i d_{\left(\mathcal{X}_{\varepsilon}^{E\left(m_{0}\right)+\hat{\delta}}, \mathcal{X}_{\varepsilon}^{E\left(m_{0}\right)-\hat{\delta}}\right)}\right) \\
& =\operatorname{cupl}\left(\mathcal{X}_{\varepsilon}^{E\left(m_{0}\right)+\hat{\delta}}, \mathcal{X}_{\varepsilon}^{E\left(m_{0}\right)-\hat{\delta}}\right) .
\end{aligned}
$$

By Proposition 6.2, that $\Psi_{\varepsilon} \circ \Phi_{\varepsilon}$ is homotopic to the inclusion

$$
j:(I \times K, \partial I \times K) \rightarrow\left(I \times K_{d_{0}}, \partial I \times K_{d_{0}}\right) .
$$

By (iii) of Proposition 7.3 ,

$$
\operatorname{cupl}\left(\Psi_{\varepsilon} \circ \Phi_{\varepsilon}\right)=\operatorname{cupl}(j) .
$$

At this point using (7.2)-(7.4) and recalling our choice of $d_{0}$, that is, Lemma 7.5 holds for $d=d_{0}$ we deduce that

$$
\operatorname{cat}\left(\mathcal{X}_{\varepsilon}^{E\left(m_{0}\right)+\hat{\delta}}, \mathcal{X}_{\varepsilon}^{E\left(m_{0}\right)-\hat{\delta}}\right) \geq \operatorname{cupl}(K)+1
$$

Thus by (7.1), $J_{\varepsilon}$ has at least $\operatorname{cupl}(K)+1$ critical points in $\mathcal{X}_{\varepsilon}^{E\left(m_{0}\right)+\hat{\delta}} \backslash$ $\mathcal{X}_{\varepsilon}^{E\left(m_{0}\right)-\hat{\delta}}$. Recalling Proposition 4.1, this completes the proof of the Theorem.

Proof of Theorem 1.1. Since $\operatorname{cupl}(K) \geq 0$ for $K \neq \emptyset$, Theorem 1.1 can be regarded as a special case of Theorem 1.4.

Proof of Remark 1.6. From Proposition 4.1 we know that the critical points $u_{\varepsilon}^{i}, i=1, \ldots, \operatorname{cupl}(K)+1$ satisfy

$$
\left\|u_{\varepsilon}^{i}(x)-\omega^{i}\left(x-x_{\varepsilon}^{i}\right)\right\|_{H^{1}} \rightarrow 0
$$

where $\varepsilon x_{\varepsilon}^{i}=\varepsilon \beta\left(u_{\varepsilon}^{i}\right)+o(1) \rightarrow x_{0}^{i} \in K$ and $\omega^{i} \in S_{m_{0}}$. Thus $w_{\varepsilon}^{i}(x)=u_{\varepsilon}^{i}\left(x+x_{\varepsilon}^{i}\right)$ converges to $\omega^{i} \in S_{m_{0}}$. Each $w_{\varepsilon}^{i}$ converges to a least energy solution of (1.8).

\section{Another proof of Theorem 1.1}

We can give another proof of Theorem 1.1 via localized mountain pass argument. We just give an outline of a proof. 


\section{Outline of a proof of Theorem 1.1 via localized mountain pass.}

Let $s_{0}>0, \nu_{*}>0$ be as in previous sections. We may assume that $0 \in K$.

It suffices to show for any $\delta \in\left(0, \nu_{*}\right)$ small that $J_{\varepsilon}(u)$ has a critical point $u \in N_{\varepsilon}(\delta)$ with $J_{\varepsilon}(u) \in\left[E\left(m_{0}\right)-\delta, E\left(m_{0}\right)+\delta\right]$ for $\varepsilon>0$ small.

Choosing $\delta>0$ smaller if necessary, as in Corollary 4.2 we can show for some $\kappa_{0}>0$ independent of $\varepsilon$ that for $\varepsilon>0$ small

$$
\left\|J_{\varepsilon}^{\prime}(u)\right\|_{H^{-1}} \geq \kappa_{0}
$$

for $u \in N_{\varepsilon}\left(\nu_{*}\right)$ with $(\widehat{\rho}(u), \varepsilon \beta(u)) \in\left([0, \delta] \times K_{d_{0}}\right) \backslash\left(\left[0, \frac{1}{2} \delta\right] \times K_{\frac{3}{4} d_{0}}\right)$ and $J_{\varepsilon}(u) \in\left[E\left(m_{0}\right)-\delta, E\left(m_{0}\right)+\delta\right]$.

If $J_{\varepsilon}(u)$ does not have any critical point with $J_{\varepsilon}(u) \in\left[E\left(m_{0}\right)-\delta, E\left(m_{0}\right)+\right.$ $\delta]$ in $N_{\varepsilon}(\delta)$, then, by Proposition [4.3, there exists $\kappa_{\varepsilon} \in\left(0, \kappa_{0}\right]$ such that

$$
\left\|J_{\varepsilon}^{\prime}(u)\right\|_{H^{-1}} \geq \kappa_{\varepsilon} \quad \text { for } u \in N_{\varepsilon}(\delta) \text { with } J_{\varepsilon}(u) \in\left[E\left(m_{0}\right)-\delta, E\left(m_{0}\right)+\delta\right] \text {. }
$$

Now we choose and fix $\omega_{0} \in S_{m_{0}}$. We set

$$
\gamma_{\varepsilon}(s)(x)=\zeta_{\frac{1}{2 \sqrt{\varepsilon}}}(|x|) \omega_{0}\left(\frac{x}{s}\right):\left[1-s_{0}, 1+s_{0}\right] \rightarrow H^{1}\left(\mathbb{R}^{N}\right) .
$$

We can easily see that

$$
\begin{aligned}
& \gamma_{\varepsilon}(s) \in N_{\varepsilon}\left(\frac{\delta}{2}\right) \quad \text { for } s \in\left[1-s_{0}, 1+s_{0}\right], \\
& \max _{s \in\left[1-s_{0}, 1+s_{0}\right]} J_{\varepsilon}\left(\gamma_{\varepsilon}(s)\right) \rightarrow E\left(m_{0}\right) \quad \text { as } \varepsilon \rightarrow 0 .
\end{aligned}
$$

If $J_{\varepsilon}(u)$ does not have any critical point with $J_{\varepsilon}(u) \in\left[E\left(m_{0}\right)-\delta, E\left(m_{0}\right)+\delta\right]$ in $N_{\varepsilon}(\delta)$, by (8.1)-(8.2), we can find a deformation flow $\eta:\left[0, T_{\varepsilon}\right] \times N_{\varepsilon}\left(\frac{\delta}{2}\right) \rightarrow$ $N_{\varepsilon}(\delta)$ such that

$$
\begin{aligned}
& \eta\left(T_{\varepsilon}, \gamma_{\varepsilon}\left(1 \pm s_{0}\right)\right)=\gamma_{\varepsilon}\left(1 \pm s_{0}\right), \\
& \left.J_{\varepsilon}\left(\eta\left(T_{\varepsilon}, u\right)\right) \leq E\left(m_{0}\right)-\frac{\widehat{\delta}}{2} \text { for } u \in N_{\varepsilon}\left(\frac{\delta}{2}\right) \text { with } J_{\varepsilon}(u) \leq E\left(m_{0}\right)+\frac{\widehat{\delta}}{2} 8.6\right)
\end{aligned}
$$

where $\widehat{\delta}=\frac{1}{2} \min \left\{\delta_{0}, \delta, \frac{\kappa_{0} \delta}{4}, E\left(m_{0}\right)-L_{m_{0}}\left(\omega\left(\frac{x}{1 \pm s_{0}}\right)\right)\right\}>0$. Here $\delta_{0}>0$ is given in Lemma 3.7

In fact, the flow $\eta(\tau, u)$ is defined as a solution of

$$
\begin{aligned}
& \frac{d \eta}{d \tau}=-\varphi(\eta) \frac{V_{\varepsilon}(\eta)}{\left\|V_{\varepsilon}(\eta)\right\|_{H^{1}}}, \\
& \eta(0, u)=u
\end{aligned}
$$


where $V_{\varepsilon}(u)$ is a pseudo-gradient vector field associated to $J_{\varepsilon}^{\prime}(u)$ and $\varphi(u)$ : $N_{\varepsilon}(\delta) \rightarrow[0,1]$ is a locally Lipschitz continuous function. With a suitable choice of $\varphi(u)$, we have

$$
\eta(\tau, u)=u \quad \text { if } J_{\varepsilon}(u) \notin\left[E\left(m_{0}\right)-2 \widehat{\delta}, E\left(m_{0}\right)+2 \widehat{\delta}\right],
$$

which implies (8.5). Moreover, we have as long as $\eta(t, u) \in N_{\varepsilon}(\delta)$ satisfies $J_{\varepsilon}(\eta(\tau, u)) \in\left[E\left(m_{0}\right)-\widehat{\delta}, E\left(m_{0}\right)+\widehat{\delta}\right]$,

$$
\begin{aligned}
\left\|\frac{d}{d \tau} \eta(\tau, u)\right\|_{H^{1}} & \leq 1 \\
\frac{d}{d \tau} J_{\varepsilon}(\eta(\tau, u)) & \leq-\frac{1}{2} \kappa_{\varepsilon}, \\
\frac{d}{d \tau} J_{\varepsilon}(\eta(\tau, u)) & \leq-\frac{1}{2} \kappa_{0} \quad \text { if } \eta(\tau, u) \in N_{\varepsilon}(\delta) \backslash N_{\varepsilon}\left(\frac{1}{2} \delta\right) .
\end{aligned}
$$

We also note that by Lemma 3.7

$$
J_{\varepsilon}(u) \geq E\left(m_{0}\right)+\delta_{0} \geq E\left(m_{0}\right)+\widehat{\delta} \text { if } u \in N_{\varepsilon}(\delta) \text { satisfies } \varepsilon \beta(u) \in K_{d_{0}} \backslash K_{\frac{3}{4} d_{0}} .
$$

For $u \in N_{\varepsilon}\left(\frac{\delta}{2}\right)$ with $J_{\varepsilon}(u) \in\left[E\left(m_{0}\right)-\widehat{\delta}, E\left(m_{0}\right)+\widehat{\delta}\right]$, we consider the flow $\eta(\tau)=\eta(\tau, u)$. As long as $\eta(\tau) \in N_{\varepsilon}(\delta)$ and $J_{\varepsilon}(\eta(\tau)) \in\left[E\left(m_{0}\right)-\widehat{\delta}, E\left(m_{0}\right)+\widehat{\delta}\right]$ hold, we have $\frac{d}{d \tau} J_{\varepsilon}(\eta(\tau)) \leq-\frac{1}{2} \kappa_{\varepsilon}$, in particular, $J_{\varepsilon}(\eta(\tau))$ decreases at a certain rate. By (8.8), if $\eta(\tau)$ leaves $N_{\varepsilon}(\delta)=\left\{u \in \widehat{S}\left(\nu_{*}\right) ; \widehat{\rho}(u)<\delta, \varepsilon \beta(u) \in\right.$ $\left.K_{d_{0}}\right\}, \eta(\tau)$ gets out through the set $\left\{u \in \widehat{S}\left(\nu_{*}\right) ; \widehat{\rho}(u)=\delta\right\}$. Therefore, by (8.7), we deduce (8.6) for large $T_{\varepsilon}$.

Thus, for sufficiently small $\varepsilon>0$, it follows from (8.4) that $\max _{s \in\left[1-s_{0}, 1+s_{0}\right]} J_{\varepsilon}\left(\gamma_{\varepsilon}(s)\right) \leq E\left(m_{0}\right)+\frac{\widehat{\delta}}{2}$. Thus $\widetilde{\gamma}_{\varepsilon}(s)=\eta\left(T_{\varepsilon}, \gamma_{\varepsilon}(s)\right)$ satisfies

$$
\begin{aligned}
& \widetilde{\gamma}_{\varepsilon}\left(1 \pm s_{0}\right)=\gamma_{\varepsilon}\left(1 \pm s_{0}\right), \\
& \max _{s \in\left[1-s_{0}, 1+s_{0}\right]} J_{\varepsilon}\left(\widetilde{\gamma}_{\varepsilon}(s)\right) \leq E\left(m_{0}\right)-\frac{\widehat{\delta}}{2} .
\end{aligned}
$$

Let $\tau_{\varepsilon}(u): N_{\varepsilon}(\delta) \subset N_{\varepsilon}\left(\nu_{*}\right) \rightarrow N_{\varepsilon}\left(5 \nu_{*}\right)$ be a map obtained in Proposition 5.1. We set

$$
\widehat{\gamma}_{\varepsilon}(s)=\tau_{\varepsilon}\left(\widetilde{\gamma}_{\varepsilon}(s)\right)
$$


Thus we have

$$
\begin{aligned}
& J_{\varepsilon}\left(\widehat{\gamma}_{\varepsilon}(s)\right) \leq J_{\varepsilon}\left(\widetilde{\gamma}_{\varepsilon}(s)\right) \leq E\left(m_{0}\right)-\frac{\widehat{\delta}}{2}, \\
& \left\|\widehat{\gamma}_{\varepsilon}(s)\right\|_{H^{1}\left(B\left(\beta\left(\widetilde{\gamma}_{\varepsilon}(s)\right), \frac{2}{\sqrt{\varepsilon}}\right)^{c}\right)} \leq \widetilde{\rho}_{\varepsilon}, \\
& \widehat{\gamma}_{\varepsilon}\left(1 \pm s_{0}\right)=\widetilde{\gamma}_{\varepsilon}\left(1 \pm s_{0}\right)=\gamma_{\varepsilon}\left(1 \pm s_{0}\right) .
\end{aligned}
$$

Here we use the fact that $\left.\widetilde{\gamma}\left(1 \pm s_{0}\right)\right|_{|x| \geq \frac{1}{\sqrt{\varepsilon}}}=\left.\gamma\left(1 \pm s_{0}\right)\right|_{|x| \geq \frac{1}{\sqrt{\varepsilon}}}=0$, which implies (8.11).

Thus $\widehat{\gamma}_{\varepsilon}(s)$ satisfies $\beta\left(\widehat{\gamma}_{\varepsilon}(s)\right) \in K_{d_{0}}$ for all $s \in\left[1-s_{0}, 1+s_{0}\right]$ and it follows from (8.9) - (8.10) that

$$
L_{m_{0}}\left(\widehat{\gamma}_{\varepsilon}(s)\right) \leq J_{\varepsilon}\left(\widehat{\gamma}_{\varepsilon}(s)\right)+o(1) \leq E\left(m_{0}\right)-\frac{\widehat{\delta}}{2}+o(1)
$$

uniformly in $s \in\left[1-s_{0}, 1+s_{0}\right]$ as $\varepsilon \rightarrow 0$.

On the other hand, by (8.11),

$$
\Lambda\left(\widehat{\gamma}_{\varepsilon}\left(1 \pm s_{0}\right)\right)=\Lambda\left(\gamma_{\varepsilon}\left(1 \pm s_{0}\right)\right)=1 \pm s_{0}+o(1) \quad \text { as } \varepsilon \rightarrow 0 .
$$

Thus for $\varepsilon>0$ small there exists $s_{\varepsilon} \in\left(1-s_{0}, 1+s_{0}\right)$ such that

$$
\Lambda\left(\widehat{\gamma}_{\varepsilon}\left(s_{\varepsilon}\right)\right)=1 \text {. }
$$

(8.12) and (8.13) contradicts with (3.7). Thus $J_{\varepsilon}(u)$ has a critical point $u \in N_{\varepsilon}(\delta)$ satisfying $J_{\varepsilon}(u) \in\left[E\left(m_{0}\right)-\delta, E\left(m_{0}\right)+\delta\right]$.

Acknowledgments. Authors started this research during the second author's visit to Dipartimento di Meccanica, Matematica e Management, Politecnico di Bari in 2016 and the first author's visit to Department of Mathematics, Waseda University in 2017. They would like to thank Politecnico di Bari and Waseda University for their kind hospitality. The first author is partially supported by INdAM-GNAMPA Project 2017 "Metodi matematici per lo studio di fenomeni fisici nonlineari". The second author is partially supported by JSPS Grants-in-Aid for Scientific Research (B) (25287025) and (B) (17H02855). 


\section{References}

[1] A. Ambrosetti, M. Badiale, S. Cingolani, Semiclassical states of nonlinear Schrödinger equations, Arch. Rational Mech. Anal. 140 (1997), no. 3, 285-300.

[2] A. Ambrosetti, P. H. Rabinowitz, Dual variational methods in critical point theory and applications, Journal of functional Analysis, 14 (1973), 349-381.

[3] H. Berestycki, P.-L. Lions, Nonlinear scalar field equations. I. Existence of a ground state, Arch. Rational Mech. Anal. 82 (1983), 313-345.

[4] A. Bahri, J. M. Coron, On a nonlinear elliptic equation involving the critical Sobolev exponent: the effect of the topology of the domain, Comm. Pure Appl. Math. 41 (1988), $253-294$

[5] T. Bartsch, T. Weth, The effect of the domain's configuration space on the number of nodal solutions of singularly perturbed elliptic equations, Topol. Methods Nonlinear Anal. 63 (2005), 109-133.

[6] V. Benci, G. Cerami, The effect of the domain topology on the number of positive solutions of nonlinear elliptic problems, Arch. Rational Mech. Anal. 114 (1991), 7993.

[7] V. Benci, G. Cerami, Multiple positive solutions of some elliptic problems via the Morse theory and the domain topology, Calc. Var. Partial Differential Equations 2 (1994), 2948.

[8] V. Benci, G. Cerami, D. Passaseo, On the number of the positive solutions of some nonlinear elliptic problems, Nonlinear Analysis, tribute in honor of G. Prodi, Quaderno Scuola Normale Sup. Pisa 1991, pp.93-107.

[9] J. Byeon, L. Jeanjean, Standing waves for nonlinear Schrödinger equations with a general nonlinearity, Arch. Ration. Mech. Anal. 185 (2007), no. 2, 185-200.

[10] J. Byeon, K. Tanaka, Semi-classical standing waves for nonlinear Schrödinger equations at structurally stable critical points of the potential, J. Euro Math. Soc., 15 (2013), 1859-1899.

[11] J. Byeon, K. Tanaka, Semiclassical standing waves with clustering peaks for nonlinear Schrödinger equations, Memoir Amer. Math. Soc., 229 (2014), 1-87.

[12] J. Byeon, K. Tanaka, Multi-bump positive solutions for a nonlinear elliptic problem in expanding tubular domains, Calc. Var. Partial Differential Equations 50 (2014), no. $1-2,365-397$.

[13] S. Cingolani, M. Clapp, S. Secchi, Multiple solutions to a magnetic nonlinear Choquard equation, Zeitschrift für Angewandte Mathematik und Physik (ZAMP), 63 (2012), 233-248. 
[14] S. Cingolani, M. Clapp, S. Secchi, Intertwining semiclassical solutions to a Schrödinger-Newton system, Discrete Continuous Dynmical Systems Series S 6 (2013), 891-908.

[15] S. Cingolani, L. Jeanjean, K. Tanaka, Multiplicity of positive solutions of nonlinear Schrödinger equations concentrating at a potential well, Calc. Var. P.D.E. 53 (2015), 413-439.

[16] S. Cingolani, L. Jeanjean, K. Tanaka, Multiple complex-valued solutions for nonlinear magnetic Schrödinger equations, J. Fixed Point Theory Appl. 19 (2017), no. 1, 37-66.

[17] S. Cingolani, M. Lazzo, Multiple semiclassical standing waves for a class of nonlinear Schrödinger equations, Topol. Methods Nonlinear Anal. 10 (1997), no. 1, 1-13.

[18] S. Cingolani, M. Lazzo, Multiple positive solutions to nonlinear Schrödinger equations with competing potential functions, J. Differential Equations 160 (2000), no. 1, 118-138.

[19] S. Cingolani, S. Secchi, M. Squassina, Semiclassical limit for Schrödinger equations with magnetic field and Hartree-type nonlinearities, Proc. Roy. Soc. Edinburgh 140 A (2010), 973-1009.

[20] M. Clapp, D. Salazar, Positive and sign changing solutions to a nonlinear Choquard equation, J. Math. Anal. Appl. 407 (2013), 1-15.

[21] M. del Pino, P. Felmer, Local mountain passes for semilinear elliptic problems in unbounded domains, Calculus of Variations and Partial Differential Equations 4 (1996), $121-137$.

[22] A. Floer, A. Weinstein, Nonspreading wave packets for the cubic Schrödinger equation with a bounded potential, J. Funct. Anal. 69 (1986), no. 3, 397-408.

[23] G. Fournier, M. Willem, Multiple solutions of the forced double pendulum equation, Ann. Inst. H. Poincaré Anal. Non Lineaire 6 (1989), suppl., 259-281.

[24] G. Fournier, M. Willem, Relative category and the calculus of variations, Variational methods, Progr. Nonlinear Differential Equations Appl. Birkhauser, Boston 4, 95-104 (1990).

[25] J. Fröhlich, E. Lenzmann, Mean-field limit of quantum Bose gases and nonlinear Hartree equation, in Séminaire: Équations aux Dérivées Partielles 2003-2004, Exp. No. XIX, 26 pp., Sémin. Équ. Dériv. Partielles, École Polytech., Palaiseau, 2004.

[26] J. Fröhlich, T.-P. Tsai, H.-T. Yau, On the point-particle (Newtonian) limit of the non-linear Hartree equation, Comm. Math. Phys. 225 (2002), 223-274.

[27] J. Hirata, N. Ikoma, K. Tanaka, Nonlinear scalar field equations in $\mathbb{R}^{N}$ : mountain pass and symmetric mountain pass approaches, Topol. Methods Nonlinear Anal. 35 (2010), no. 2, 253-276. 
[28] L. Jeanjean, K. Tanaka, A remark on least energy solutions in $\mathbb{R}^{N}$, Proc. Amer. Math. Soc. 131, 8, (2003) 2399-2408.

[29] E. Lenzmann, Uniqueness of ground states for pseudorelativistic Hartree equations, Analysis and PDE, 2 (2009), 1-27.

[30] E. H. Lieb, Existence and uniqueness of the minimizing solution of Choquard's nonlinear equation, Stud. Appl. Math. 57 (1977), 93-105.

[31] E. H. Lieb, Sharp constants in the Hardy-Littlewood-Sobolev and related inequalities, The Annals of Mathematics 118 (1983), 349-374.

[32] E. H. Lieb, M. Loss, "Analysis", Graduate Studies in Math. 14, Amer. Math. Soc. 1997.

[33] P.-L. Lions, The Choquard equation and related questions, Nonlinear Anal. T.M.A. 4 (1980), 1063-1073.

[34] L. Ma, L. Zhao, Classification of positive solitary solutions of the nonlinear Choquard equation, Arch. Rational Mech. Anal. 195 (2010), 455-467.

[35] I. M. Moroz, R. Penrose, P. Tod, Spherically-symmetric solutions of the SchrödingerNewton equations, Topology of the Universe Conference (Cleveland, OH, 1997), Classical Quantum Gravity 15 (1998), 2733-2742.

[36] V. Moroz, J. Van Schaftingen, Groundstates of nonlinear Choquard equations: existence, qualitative properties and decay asymptotics, J. Funct. Anal. 265 (2013), no. 2, $153-184$.

[37] V. Moroz, J. Van Schaftingen, Existence of groundstates for a class of nonlinear Choquard equations, Trans. Amer. Math. Soc. 367 (2015), 6557-6579.

[38] V. Moroz, J. Van Schaftingen, Semi-classical states for the Choquard equations, Calc. Var. Partial Differential Equations, 52 (2015), 199-235.

[39] V. Moroz, J. Van Schaftingen, A guide to the Choquard equation, to appear in J. Fixed Point Theory (2016)

[40] Y.-G. Oh, Existence of semiclassical bound states of nonlinear Schrödinger equations with potentials of the class $(V)_{a}$, Comm. Partial Differential Equations 13 (1988), no. $12,1499-1519$.

[41] S. Pekar, Untersuchung über die Elektronentheorie der Kristalle, Akademie Verlag, Berlin, 1954.

[42] R. Penrose, On gravity's role in quantum state reduction, Gen. Rel. Grav. 28 (1996), 581-600. 
[43] R. Penrose, Quantum computation, entanglement and state reduction, R. Soc. Lond. Philos. Trans. Ser. A Math. Phys. Eng. Sci. 356 (1998), 1927-1939.

[44] R. Penrose, "The road to reality. A complete guide to the laws of the universe", Alfred A. Knopf Inc., New York (2005).

[45] S. Secchi, A note on Schrödinger-Newton systems with decaying electric potential, Nonlinear Analysis 72 (2010), 3842-3856.

[46] E. H. Spanier, "Algebraic topology", Corrected reprint, Springer-Verlag, New YorkBerlin, 1981, xvi+528.

[47] P. Tod, The ground state energy of the Schrödinger-Newton equation, Physics Letters A 280 (2001), 173-176.

[48] M. Yang, J. Zhang, Y. Zhang, Multi-peak solutions for nonlinear Choquard equation with a general nonlinearity, Comm. Pure Appl. Anal. 16 (2017), 493-512.

[49] J. Wei, M. Winter, Strongly interacting bumps for the Schrödinger-Newton equation, J. Math. Phys. 50 (2009), 012905. 